\title{
Systems for Knowledge and Belief
}

\author{
WIEBE VAN DER HOEK, Free University Amsterdam, Department of \\ Mathematics and Computer Science, De Boelelaan 1081, NL-1081 HV \\ Amsterdam, The Netherlands. E-mail: wiebe@cs.vu.nl
}

\begin{abstract}
We investigate modal systems for knowledge and belief, taking as a starting point a logic that was originally introduced by Kraus and Lehmann. We derive several properties and discuss (their) consequences for the epistemic operators. Kraus and Lehmann observed that adding the axiom $B_{1} \varphi \rightarrow B_{1} K_{1} \varphi$ to the system gives a collapse of knowledge and belief: $\left(K_{i} \varphi \leftrightarrow B_{i} \varphi\right)$. We investigate the cause(s) of this problem and suggest a 'simlar' system that does allow the same axiom without the mentioned collapse. We consider as the main benefit of this paper, however, the techniques that are developed to come to this solution. It appears that applying basic correspondence theory to a multi-modal system allows a systematic examination of possible combinations of epistemic operators.
\end{abstract}

Keywords: Combined epistemic and doxastic logic, positive and negative introspection and extraspection, multi-modal logic, correspondence theory.

\section{Introduction}

We discuss (multi modal) logics for both knowledge and belief which are to be interpreted on Kripke structures. The basic system for our discussion is introduced by Kraus and Lehmann [19]. Knowledge $(K)$ and belief $(B)$ are both interpreted (as necessity operators with respect to two binary relations) over Kripke structures. (For an introduction to modal logic, see [4] or [18].) We denote their basic system with $K B_{C D}$. What is interesting in $K B_{C D}$ is, that it does not only give notions of knowledge and belief (which on their own are rather familiar ones-see [9] or $[23,24]$ ), but also some interaction properties between the two (an alternative approach to have both notions in one system is to define one in terms of the other-cf. [21] or [27]).

In the literature of philosophical logic, systems for knowledge and belief were studied in the 1960 s (cf. [10]). In the 1980s, these notions became one of the central themes in the field of $\mathrm{AI}[9]$ and are thus gaining their place in the field of computer science $[23,24]$. It now seems conventional to take the system $S 5$ for knowledge and weak $S 5$ (or KD45) for belief (cf. $[9,10,23,24])$. To be more precise, it is customary to ascribe the following properties to belief. One does not believe false assertions $(\neg B \perp)$, believers have positive- $(B \varphi \rightarrow B B \varphi)$ as well as negative introspection $(\neg B \varphi \rightarrow B \neg B \varphi)$. Knowledge should moreover also be veridical: $(K \varphi \rightarrow \varphi)$. We will give a rather systematic classification of properties like these in Section 6 . To mould the notions into a logical system, one usually adds the inference rules modus ponens $(\vdash \varphi, \vdash \varphi \rightarrow \psi \Rightarrow \vdash \psi)$ and necessitation for $K$ as well as $B(\vdash \varphi \Rightarrow \vdash K \varphi, \vdash \varphi \Rightarrow \vdash B \varphi)$.

Once we have given the basic system $K B_{C D}$ for knowledge and belief, we investigate some of its properties. One important theme in this paper is a problem that is also mentioned in [19]. It appears that adding the axiom $(B \varphi \rightarrow B K \varphi)$ to $K B_{C D}$ yields $(K \varphi \leftrightarrow B \varphi)$, which is clearly undesirable. We will develop some techniques (in Section 4 ) to study this problem systematically, and suggest some solutions. These techniques are presented in a slightly more general setting than needed for this problem only, but the generalizations are obtained in a very natural way. 
In Section 2 we introduce $K B_{C D}$ and show the system in action by deriving some theorems. We will briefly discuss the impact of some of the properties of $K B_{C D}$ on the notions of knowledge and belief. In Section 3 we give a Kripke semantics for a 'finitary part' of the logic, and prove completeness: in particular, we will construct a canonical model; this construction will be used throughout the paper to obtain completeness results for modified systems as well. In Section 4 we systematically investigate the impact of particular axioms on the canonical models (for those axioms). It will appear, that these correspondences are not hard to prove, but are, at the same time, easily transferable to more specific cases. It gives us an alternative way to derive $K B_{C D^{-}}$ theorems, and also enables us to prove that some formulas are not theorems.

In Section 5 we discuss the problem that we mentioned above: adding $(B \varphi \rightarrow B K \varphi)$ to $K B_{C D}$ yields $(K \varphi \leftrightarrow B \varphi)$. In Section 6, properties like positive and negative introspection (and 'extraspection') are introduced. From Section 4 we know how these properties are related with the Kripke structure, so that we can investigate which properties $K B_{C D}$ does not have. It will turn out that $K B_{C D}$ is 'saturated' with respect to introspection and extraspection properties: adding any of them to $K B_{C D}$ yields $(K \varphi \leftrightarrow B \varphi)$. We show how one can define systems for knowledge and belief with various degrees of introspection, without having $(K \varphi \leftrightarrow B \varphi)$. In Section 7 we give some conclusions.

\section{$2 K B_{C D}$ as a basis for knowledge and belief}

Kraus and Lehmann [19] introduced a system (which we will denote with $K B_{C D}$ ) that can deal with knowledge and belief simultaneously. They used $2 n$ operators $K_{1}, \ldots, K_{n}, B_{1}, \ldots, B_{n}$, modelling the knowledge and belief of $n$ agents from an index set People $=\{1, \ldots, n\}$. In general, given a set $P$ of propositional atoms and $O$ of operators, a language $L(P, O)$ is the smallest set $S \supseteq \mathrm{P}$ which is closed both under infix attachment of $\wedge, \vee, \rightarrow$, and $\leftrightarrow$, and prefix placing of $\neg$ and operators $O \in \mathrm{O}$. For the moment, our language $\mathrm{KB} C D$ for $K B_{C D}$ is $\mathrm{L}(\mathrm{P}, \mathrm{O})$, where $\mathrm{P}$ is a set of atoms and $\mathrm{O}=\left\{C, D, E, F, K_{i}, B_{i} \mid i \leq n\right\}$. If $|\mathrm{O}|>1$, we say to have a multi modal logic. In the sequel, if we write $K_{2}$ or $B_{i}, i$ is a member of People. The system $K B_{C D}$ has four levels, the first of which is a propositional one:

(A0) Any axiomatization of the propositional calculus

(R0) $\vdash \varphi, \vdash \varphi \rightarrow \psi \Rightarrow \vdash \psi$.

Next, there is a level concerning properties of knowledge $\left(K_{i}\right)$ and common knowledge $(C)$. $E \varphi$ (everybody knows that $\varphi$ ) is defined as follows: $E \varphi \equiv K_{1} \varphi \wedge K_{2} \varphi \wedge \ldots \wedge K_{n} \varphi$. $C \varphi$ is supposed to mean $(E \varphi \wedge E E \varphi \wedge \ldots)$. Somewhat surprisingly, this infinite conjunction can be axiomatized.

$$
\begin{array}{ll}
\text { (A1) } & K_{i}(\varphi \rightarrow \psi) \rightarrow\left(K_{i} \varphi \rightarrow K_{i} \psi\right) \\
\text { (A2) } & K_{i} \varphi \rightarrow \varphi \\
\text { (A3) } \neg K_{i} \varphi \rightarrow K_{i} \neg K_{i} \varphi \\
\text { (A4) } C(\varphi \rightarrow \psi) \rightarrow(C \varphi \rightarrow C \psi) \\
\text { (A5) } C \varphi \rightarrow E \varphi \\
\text { (A6) } C \varphi \rightarrow E C \varphi \\
\text { (A7) } C(\varphi \rightarrow E \varphi) \rightarrow(\varphi \rightarrow C \varphi) \\
\text { (R1) } \vdash \varphi \Rightarrow \vdash C \varphi .
\end{array}
$$

Then, a level concerning general properties about belief $\left(B_{i}\right)$, and common belief $(D) . F \varphi$ 
(everybody believes that $\varphi$ ) is defined as follows: $F \varphi \equiv B_{1} \varphi \wedge B_{2} \varphi \wedge \ldots \wedge B_{n} \varphi . D \varphi$ is supposed to be the infinite conjunction $(F \varphi \wedge F F \varphi \wedge \ldots)$.

$$
\begin{array}{ll}
\text { (A8) } & B_{i}(\varphi \rightarrow \psi) \rightarrow\left(B_{i} \varphi \rightarrow B_{\imath} \psi\right) \\
\text { (A9) } & \neg B_{2} \text { false } \\
\text { (A10) } & D(\varphi \rightarrow \psi) \rightarrow(D \varphi \rightarrow D \psi) \\
\text { (A11) } & D \varphi \rightarrow F \varphi \\
\text { (A12) } & D \varphi \rightarrow F D \varphi \\
\text { (A13) } & D(\varphi \rightarrow F \varphi) \rightarrow(F \varphi \rightarrow D \varphi)
\end{array}
$$

Finally, there is a level combining (common) knowledge and (common) belief:

$$
\begin{array}{ll}
\text { (A14) } & K_{i} \varphi \rightarrow B_{i} \varphi \\
\text { (A15) } & B_{i} \varphi \rightarrow K_{i} B_{\imath} \varphi \\
\text { (A16) } & C \varphi \rightarrow D \varphi .
\end{array}
$$

\section{LEMMA 2.1}

Let $\left[{ }^{\alpha} / \beta\right] \varphi$ be any formula, which arises from $\varphi$ by substituting any occurrence(s) of $\beta$ in $\varphi$ by $\alpha$. Then the following rule of substitution Sub is derivable in $K B_{C D}$

$$
\text { Sub } \vdash \alpha \leftrightarrow \beta \Rightarrow \vdash \varphi \leftrightarrow\left[{ }^{\alpha} / \beta\right] \varphi .
$$

PROOF. Here, we omit the simple, but tedious proof by induction on the complexity of $\varphi$, which should be preceded by an inductive definition of substitution.

The following theorem shows that the notions of knowledge and belief, as defined in $K B_{C D}$ (and considered separately) have at least the properties of those in $S 5$ and weak $S 5$, respectively (cf. the introduction, or $[9,23,24])$.

LEMMA 2.2

In the system $K B_{C D}$, knowledge $\left(K_{i}\right)$ has all the properties of $S 5$ whereas belief $\left(B_{\imath}\right)$ has those of weak $S 5$.

PROOF. Modus ponens is immediate from R0. Also, R1, $(\vdash \varphi \Rightarrow \vdash C \varphi$ ), together with A5 $(\vdash C \varphi \rightarrow E \varphi)$ and the definition of $E\left(\equiv K_{1} \varphi \wedge \ldots \wedge K_{n} \varphi\right)$ gives necessitation for $K_{i}\left(\vdash \varphi \Rightarrow \vdash K_{i} \varphi\right)$. Axiom A14 $\left(K_{i} \varphi \rightarrow B_{i} \varphi\right)$ then yields necessitation for $B_{i}$ as well. Veridicality and negative introspection are explicitly added for $K_{2}$ to $K B_{C D}$ (A2 and A3, respectively). Positive introspection for $K_{i}$ follows from A2 and $\mathrm{A} 3$ ( $K_{i} \varphi \Rightarrow A 2$ $\left.\neg K_{i} \neg K_{i} \varphi \Rightarrow_{A 3} K_{i} \neg K_{i} \neg K_{i} \varphi \Rightarrow_{A 3} K_{i} K_{\imath} \varphi\right)$. Thus we have that knowledge in $K B_{C D}$ is ' $S 5$-like'. Concerning belief, to show that this is 'weak $S 5$-like', since we have A9, we only have to derive the two introspection properties for $B_{\boldsymbol{i}}$. Positive introspection follows immediately from A15 $\left(B_{i} \varphi \rightarrow K_{i} B_{i} \varphi\right)$ and $\mathrm{A} 14\left(K_{i} \psi \rightarrow B_{i} \psi\right)$. Finally we prove negative introspection for $B_{i}$ :
1. $\quad B_{\imath} \varphi \leftrightarrow K_{i} B_{i} \varphi$
2. $\quad \neg B_{i} \varphi \leftrightarrow \neg K_{2} B_{i} \varphi$
' $\leftarrow$ ': A2, ' $\rightarrow$ ': A15
$\neg B_{i} \varphi \leftrightarrow \neg K_{2} B_{i} \varphi$
$\neg K_{i} B_{i} \varphi \rightarrow K_{i} \neg K_{i} B_{i} \varphi$
$\mathrm{A} 0,1$
$\begin{array}{ll}\text { 3. } & \neg K_{i} B_{i} \varphi \rightarrow K_{i} \neg K_{i} B_{i} \varphi \\ \text { 4. } & \neg B_{i} \varphi \rightarrow K_{i} \neg K_{i} B_{i} \varphi\end{array}$
5. $\quad \neg B_{i} \varphi \rightarrow K_{i} \neg B_{i} \varphi$
6. $\left.\quad K_{i}\right\urcorner B_{i} \varphi \rightarrow B_{i} \neg B_{2} \varphi$
A0, 2, 3
7. $\quad \neg B_{i} \varphi \rightarrow B_{i} \neg B_{i} \varphi$
Sub (subst of $B_{i} \varphi$ for $K_{\imath} B_{i} \varphi(1)$ in 4) 


\section{Systems for Knowledge and Belief}

\section{DEFINTTION 2.3}

We say that an operator $\square$ is a (normal) modal operator (in $L$ ) if it satisfies:

(i) $\vdash \varphi \Rightarrow \vdash \square \varphi$ necessitation

(ii) $\vdash \square(\varphi \rightarrow \psi) \rightarrow(\square \varphi \rightarrow \square \psi) \quad$ distribution

Moreover, we call a modal logic $L$ normal if it contains A0, R0, necessitation and distribution.

\section{LEMMA 2.4}

The operators $K_{i}, B_{i}, C, D, E$ and $F$ are all normal modal operators in $K B_{C D}$.

REMARK 2.5

The observation above immediately follows from the definition of $E$ and $F$ and the axioms of $K B_{C D}$. This implies that we may apply our modal intuitions to derive several properties of our operators. To mention some, we have $\vdash \varphi \rightarrow \psi \Rightarrow \vdash \square \varphi \rightarrow \square \psi(i), \vdash \square(\varphi \wedge \psi) \leftrightarrow(\square \varphi \wedge \square \psi)$ (ii) and $\vdash(\square \varphi \vee \square \psi) \rightarrow \square(\varphi \vee \psi)$ (iii). When we want to use such properties for $\square$ (e.g. when deriving some $K B_{C D}$-theorems (2.8)), we refer to them as 2.5 . These properties naturally provide some attributes for the epistemic operators they are supposed to model; for a discussion we refer to [13].

THEOREM 2.6

In [19], it is claimed (not proven) that $K B_{C D}$ has the following theorems:

$$
\begin{aligned}
& K_{i} \neg \varphi \rightarrow \neg B_{i} \varphi \\
& B_{i} \varphi \leftrightarrow K_{i} B_{i} \varphi \\
& \neg B_{i} \varphi \leftrightarrow K_{i} \neg B_{i} \varphi \\
& K_{i} \varphi \leftrightarrow B_{i} K_{i} \varphi \\
& \neg K_{i} \varphi \leftrightarrow B_{i} \neg K_{i} \varphi \\
& B_{i} \varphi \leftrightarrow B_{2} B_{i} \varphi \\
& \neg B_{i} \varphi \leftrightarrow B_{i} \neg B_{i} \varphi
\end{aligned}
$$

$$
\begin{aligned}
& B_{i}\left(B_{\mathfrak{i}} \varphi \rightarrow \varphi\right) \\
& D \varphi \leftrightarrow D F \varphi \\
& D \varphi \leftrightarrow F D \varphi \\
& F D \varphi \leftrightarrow D F D \varphi \\
& D \varphi \leftrightarrow D D \varphi \\
& C(\varphi \wedge \psi) \leftrightarrow C \varphi \wedge C \psi \\
& D(\varphi \wedge \psi) \leftrightarrow D \varphi \wedge D \psi
\end{aligned}
$$

REMARK 2.7

Where in this logic, knowledge and belief are defined as separate entities with some interaction (A14-A 16) axioms, an alternative approach is to take one of the two as basic, and connect the two in one fundamental definition. A popular direction follows the slogan "knowledge = justified, true belief' (already advocated in the 1960s by e.g. [21]), but an opposite view is taken in [27], where belief (or rather $B\left(\varphi, \varphi_{a s s}\right)$, the belief in $\varphi$ relative to some 'unusuality assertion' $\varphi_{a s s}$ ) is defined in terms of knowledge. In [27] it is shown that, when $S 5$ is taken for knowledge, the $K D 45$-properties for belief follows from their fundamental definition! The same even holds for the interaction axioms $\mathrm{A} 14$ and $\mathrm{A} 15$ of $K B_{C D}$ and the theorems T1-T7 (T8 can be shown to be also valid in their approach). However, from their proofs it follows that when the $B$-operator occurs more then once in a theorem, it is assumed that all the unusuallity assertions are the same. For example, one can derive in their system $B\left(B\left(\varphi, \varphi_{a s s}\right), \varphi_{a s s}\right) \leftrightarrow B\left(\varphi, \varphi_{a s s}\right)$ (cf. T8), but not $\left.\left(B\left(B \varphi, \varphi_{a s s}\right),(B \varphi)_{a s s}\right)\right) \leftrightarrow B\left(\varphi, \varphi_{a s s}\right)$. More generally, as is also stated in [27], it is not always clear which choice should be made for $\varphi_{\text {ass. }}$.

To see our system $K B_{C D}$ in action, we provide a derivation for T8. For a proof of the other theorems of 2.6, we refer to [13].

PROPOSITION 2.8

The following proves $\mathrm{T} 8$ : 
ProOF.

1. $\quad B_{i} \varphi \leftrightarrow K_{i} B_{i} \varphi$

2. $\quad \neg K_{i} B_{i} \varphi \rightarrow K_{i} \neg K_{i} B_{i} \varphi$

3. $\quad \neg B_{i} \varphi \rightarrow K_{i} \neg K_{i} B_{i} \varphi$

$\mathrm{A} 0,1,2$

4. $\quad \neg B_{i} \varphi \rightarrow K_{i} \neg B_{1} \varphi$

(1) in 3)

5. $\quad \neg B_{i} \varphi \rightarrow B_{i} \neg B_{i} \varphi$

A14, 4

$\left(\neg B_{i} \varphi \vee B_{i} \varphi\right) \rightarrow\left(B_{i} \neg B_{\imath} \varphi \vee B_{\imath} \varphi\right)$

A0, 5

7. $\quad\left(B_{2} \neg B_{i} \varphi \vee B_{i} \varphi\right) \rightarrow B_{2}\left(\neg B_{i} \varphi \vee \varphi\right)$

8. $\quad B_{\mathfrak{\imath}}\left(\neg B_{i} \varphi \vee \varphi\right) \rightarrow B_{1}\left(B_{i} \varphi \rightarrow \varphi\right)$

A0, 2.5

9. $\quad\left(\neg B_{\imath} \varphi \vee B_{i} \varphi\right) \rightarrow B_{i}\left(B_{i} \varphi \rightarrow \varphi\right)$

$\mathrm{A} 0,6,7,8$

10.

$B_{z}\left(B_{i} \varphi \rightarrow \varphi\right)$

9, A0, R0

Note how first negative introspection for $B_{\mathbf{i}}$ is derived (5), which then immediately (using only propositional logic and modal observations for $B_{i}$ ) yields the result. We will later also argue semantically (as a consequence of 4.5 ), that 10 follows directly from 5 .

We mentioned already in the introduction that one typical property that distinguishes knowledge from belief is that knowledge is veridical, i.e. known facts are true. Although this property does not hold for belief, T8 expresses that agent $i$ believes that it does hold; $B_{i}\left(B_{i} \varphi \rightarrow \varphi\right)$. Note that $\mathrm{T} 8$ implies that, by definition of $F$, we also have $\vdash B_{2}(F \varphi \rightarrow \varphi)$. Since this is true for arbitrary $i \in$ People, we have

$$
\vdash F(F \varphi \rightarrow \varphi)
$$

expressing that everybody believes that 'the belief of everybody' is also veridical. In the system $K B_{C D}$, knowledge is stronger than belief, which is expressed by $\mathrm{A} 14, K_{\imath} \varphi \rightarrow B_{i} \varphi$. A14 seems perfectly reasonable ${ }^{1}$ (but cf. also [28]). Of course, one does not want knowledge and belief to collapse, so in particular, we do not want $\mathrm{A} 14^{\prime}: B_{i} \varphi \rightarrow K_{\imath} \varphi$. For one class of formulas, however, belief and knowledge are the same.

\section{DEFINITION 2.9}

A formula with occurrences of $K_{i}$ or $B_{i}$ is called an epistemic formula. The belief set (knowledge set) of an agent $i$ in a system $L$ is defined as $\left\{\varphi \mid L \vdash B_{i} \varphi\right\}\left(\left\{\varphi \mid L \vdash K_{i} \varphi\right\}\right)$. A formula $\varphi$ is $i$-doxastic sequenced if there are $\psi$, operators $X_{1}, \ldots X_{n} \in\left\{K_{1}, B_{i}, \neg K_{2}, \neg B_{i}\right\}$ and $n>0$ such that $\varphi=X_{1} X_{2} \ldots X_{n} \psi$. We will not always mention reference to agent $i$.

THEOREM 2.10

For any $i$-doxastic sequenced $\varphi$, we have:

$K B_{C D} \vdash\left(K_{i} \varphi \leftrightarrow \varphi\right) \wedge\left(\varphi \leftrightarrow B_{\imath} \varphi\right)$.

PROOF. Immediate from A2 and A3, combined with 2.2 and T2-T7 of 2.6.

\section{COROLLARY 2.11}

For all $i$-doxastic sequenced $\varphi$ :

$K B_{C D} \vdash \varphi \Leftrightarrow K B_{C D} \vdash K_{i} \varphi \Leftrightarrow K B_{C D} \vdash B_{i} \varphi$

Theorem 2.10 implies that in $K B_{C D} i$-doxastic sequenced formulas are believed by agent $i$ iff they are known by agent $i$. Thus, knowledge and belief do collapse for believed facts and for

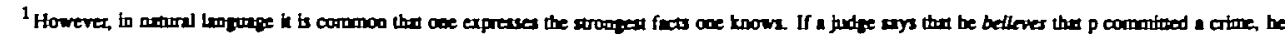

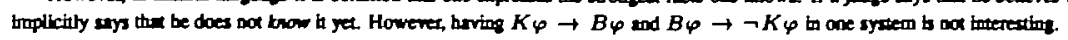


facts $\varphi$ for which $\neg K_{i} \varphi$ holds. In particular, $B_{i} K_{i} \varphi \rightarrow K_{i} K_{i} \varphi$ is valid. The following theorem shows that the formulas of 2.9 can be reduced to a formula with at most one main epistemic operator, provided that all epistemic operators have the same subscript. It implies that $K B_{C D}$ is 'optimally manageable': all sequences of operators and $\neg \mathrm{s}$ can be rewritten to a sequence with at most one operator. So, if $K B_{C D}$ models 'our' knowledge and belief, in every-day-life we never need to use complicated 'epistemic phrases' like 'I believe, that I know to believe ...'.

THEOREM 2.12

Let $i$ be given, $1 \leq i \leq n$. Let $X, Y \in\left\{K_{i}, B_{i}, \neg\right\}$ such that $Y \neq \neg$ and let $\bar{X}$ be a sequence of $X$ 's. Let $\varphi$ be any $K B_{C D}$ - formula. Then $K B_{C D} \vdash \bar{X} Y \varphi \leftrightarrow(\neg) Y \varphi$, where the ' $\neg$ ' is present if the number of ' $\neg$ ' in $\bar{X}$ is odd.

PROOF. Immediate from 2.10.

\section{Kripke semantics for $\mathrm{KB}$}

In this section we introduce a semantics for $K B_{C D}$-like systems. Unlike the completeness proof of [19], which is based on the construction of a 'universal model' using labelled traces, we will construct a 'canonical model' for any consistent formula $\varphi$, thus applying ideas from classical modal logic (cf. $[4,18]$ ). To emphasize that the structure of a model for a particular system heavily depends on the specific axioms of that system, we start out with a kind of 'barest' model. Moreover, since in this paper the notions of knowledge and belief (and their interactions) are our primary concern, we start out by simplifying $K B_{C D}$ : in the sequel, we will not consider 'common knowledge' or 'common belief' any longer. This enlightens our considerations on completeness substantially (cf. $3.12,3.15$ ).

DEFINITION 3.1

The system $K B$ is a logic in the language $\mathrm{KB}=\mathrm{L}\left(\mathrm{P},\left\{K_{\imath}, B_{i}, E, F\right\}\right)$. It consists of the axioms $\mathrm{A} 0-\mathrm{A} 3, \mathrm{~A} 8-\mathrm{A} 9$, and A14-A15. As inference rules it has R0 and Necessitation for $K$. From now, we will use ' - ' and ' $\vdash_{K B}$ ' interchangeably.

LEMMA 3.2

For any $\varphi \in \mathrm{KB}, \vdash_{K B} \varphi$ iff $\vdash_{K B_{C D}} \varphi$.

As a consequence of 3.2 we know that the theorems T1-T8 are derivable in $K B$.

DEFINITION 3.3

A Kripke model $\mathcal{M}$ for a modal language $\mathrm{L}$ with one modal operator $\square$ is a tuple $\langle W, R, \pi\rangle$, where $W$ is a set of worlds, $R \subseteq W \times W$ a binary relation, and $\pi: W \rightarrow P \rightarrow\{$ true, false $\}$ a truth assignment to the propositional atoms for each world $w \in W$. Truth definition for $\varphi \in L$ at $w$, written $(\mathcal{M}, w) \vDash \varphi$, is:

1. $(\mathcal{M}, w) \models p$ iff $\pi(w)(p)=$ true

2. $(\mathcal{M}, w) \vDash \psi \wedge \chi$ iff $(\mathcal{M}, w) \vDash \psi$ and $(\mathcal{M}, w) \vDash \chi$

3. $(\mathcal{M}, w) \vDash \neg \psi$ iff not $(\mathcal{M}, w) \vDash \psi$

4. $(\mathcal{M}, w) \models \square \psi$ iff for all $v$ for which $R_{i} w v,(\mathcal{M}, v) \models \psi$.

We say that an operator that is defined like $\square$ for $R$, is a necessity operator for $R$. For any modal operator $\square$, we define $\square=\neg \square \neg$. $\square$ is called the possibility operator for $R$. We then say that $\varphi$ is satisfiable if $\varphi$ is true at some world $w$ in some model $\mathcal{M}, \varphi$ is true in model $\mathcal{M}$ $(\mathcal{M} \models \varphi)$ if $(\mathcal{M}, w) \vDash \varphi$ for all worlds of $\mathcal{M}$, and, finally, $\varphi$ is valid $(\models \varphi)$ if it is true in all models. For any class $\mathcal{C}$ of models, we write $\models_{\mathcal{C}} \varphi$ if $\varphi$ is true in all models in $\mathcal{C}$. 
As is easily verified, we have $(\vDash \varphi \Rightarrow \vDash \square \varphi)$ and $(\vDash \square(\varphi \rightarrow \psi) \rightarrow(\square \varphi \rightarrow \square \psi))$. Since also all propositional tautologies and modus ponens are valid, this explains why Kripke structures are so suitable for interpreting modal formulas: necessitation and distribution are valid. To summarize, we have the following ( $\mathrm{K}$ is the 'minimal' normal modal logic).

\section{LEMMA 3.4}

For all $\varphi \in L, \vdash_{K} \varphi \Rightarrow \vDash \varphi$.

The proof of the converse, (which is equivalent to saying that $\mathrm{K}$-consistent formulas are satisfiable (in some Kripke model)) is also a fact from the modal logic folklore. However, for future reference, we will sketch the idea of the proof (and the construction of the model). This construction is known as the Henkin construction, and takes full benefit of the similarity between properties of maximal consistent sets on the syntactic side (3.5) and the truth definition of formulas in a world on the model-theoretic side (3.3). We only give a short sketch here, the reader is referred to $[4,18]$ or [7] for further details. We start out by repeating the notion of maximal consistent sets.

A set $\Phi$ is maximal consistent (m.c.) in a logic $L$ if it is: (i) consistent (in $L$ ) and (ii) for all $\varphi, \Phi \cup\{\varphi\}$ is consistent $\Rightarrow \varphi \in \Phi$. Due to a theorem of Lindenbaum (cf. [3]), such maximal consistent sets do exist for the logic $K B$ and its variants that we discuss here. Moreover, each consistent formula $\varphi$ is contained in a m.c. set. We assume familiarity with m.c. sets (cf. $[4,18]$ ), but summarize their vital properties in Lemma 3.5. Then we proceed by giving the definition of the canonical model for a (normal) modal logic (3.6) and recall some of its properties in 3.7. These notions and results will be needed in the sequel.

\section{LEMMA 3.5}

Let $\mathrm{L}$ be any normal modal logic (cf. 2.3). Then:

1. Every L-consistent set $\Phi$ can be extended to a m.c. set $\Sigma$

2. Suppose $\Sigma$ is m.c. in L. Then:

(a) $\varphi \notin \Sigma \Leftrightarrow \neg \varphi \in \Sigma$

(b) $(\varphi \wedge \psi) \in \Sigma \Leftrightarrow \varphi \in \Sigma$ and $\psi \in \Sigma$

(c) $(\varphi \vee \psi) \in \Sigma \Leftrightarrow \varphi \in \Sigma$ or $\psi \in \Sigma$

(d) $\Phi \vdash_{L} \varphi$ iff $\Sigma \vdash_{L} \varphi$ for every m.c. set $\Sigma \supseteq \Phi$.

DEFINITION 3.6

The canonical model $\mathcal{M}^{c}=\left\langle W^{c}, R^{c}, \pi^{c}\right\rangle$ for a modal logic $\mathrm{L}$ is defined as follows:

- $W^{c}=\{\Sigma \mid \Sigma$ is a maximal $L$-consistent set $\}$

- $R^{c}=\{(\Sigma, \Delta) \mid$ for all $\square \varphi \in \Sigma \Rightarrow \varphi \in \Delta\}$

- $\pi^{c}(\Sigma)(p)=$ true iff $p \in \Sigma$.

LEMMA $3.7([7,18])$

For all $\varphi$ and m.c. sets $\Sigma \in \mathcal{M}^{c}$ :

- $\square \varphi \in \Sigma \Leftrightarrow \forall \Delta \in \mathcal{M}^{c}\left(R^{c} \Sigma \Delta \Rightarrow \varphi \in \Delta\right)$

- $\varrho \varphi \in \Sigma \Leftrightarrow \exists \Delta \in \mathcal{M}^{c}\left(R^{c} \Sigma \Delta \wedge \varphi \in \Delta\right)$

- $R^{c} \Gamma \Delta \Leftrightarrow$ for all $\varphi:(\varphi \in \Delta \Rightarrow \square \varphi \in \Gamma)$

LEMMA 3.8 ('fundamental theorem' $[4,18]$ )

$(\mathcal{M}, \Sigma) \vDash \varphi$ iff $\varphi \in \Sigma$. 


\section{Systems for Knowledge and Belief}

PROOF. For atomic formulas, this is immediate from the definition of $\pi^{c}$. For conjunctions and negations it follows from 3.5.(2a) and 3.5.(2b), respectively. If $\varphi=\square \psi:(\mathcal{M}, \Sigma) \vDash \square \psi$ iff (by 3.3) for all $\Delta$ with $R^{c} \Sigma \Delta,(\mathcal{M}, \Delta) \vDash \psi$ iff (by induction) for all $\Delta$ with $R^{c} \Sigma \Delta, \psi \in \Delta$ iff (3.7) $\square \varphi \in \Sigma$.

\section{COROLLARY 3.9}

$\vdash_{K} \varphi$ iff $\vDash \varphi$.

PROOF. The 'only if' part is 3.4. For the 'if' part, suppose $\nvdash_{K} \varphi$, i.e., $\neg \varphi$ is K-consistent. Then, by $3.5 .1,\{\neg \varphi\}$ is contained in a m.c. set $\Sigma$. By $3.8,\left(\mathcal{M}^{c}, \Sigma\right) \models \neg \varphi$, implying $\not \models$.

Now we start to rig our bare model to models for $K B$. Of course we have to add a number of binary relations, so that our $K B$-models will be tuples

$$
\left\langle W, \pi, S_{1}, \ldots, S_{n}, S_{E}, T_{1}, \ldots, T_{n}, T_{F}\right\rangle,
$$

where $S_{2}$ is the relation for $K_{2}, T_{i}$ for $B_{i}, S_{E}$ for $E$, and $T_{F}$ for $F$, respectively. More interestingly, we will see that the axioms of $K B$ force special properties upon those relations (in the canonical model).

EXAMPLE 3.10

As an easy example, consider the axiom $K_{2} \varphi \rightarrow B_{2} \varphi$. In $\mathcal{M}^{c}$ this leads to: $T_{i}^{c} \Gamma \Delta \Leftrightarrow\left\{\varphi \mid B_{i} \varphi \in\right.$ $\Gamma\} \subseteq \Delta \Rightarrow\left\{\varphi \mid K_{i} \varphi \in \Gamma\right\} \subseteq \Delta \Leftrightarrow S_{2}^{c} \Gamma \Delta$.

DEFINITION 3.11

A $K B$-model $\mathcal{M}$ is a tuple $\left\langle W, \pi, S_{1}, \ldots, S_{n}, S_{E}, T_{1}, \ldots, T_{n}, T_{F}\right\rangle$ satisfying:

1. $S_{i}$ is an equivalence relation (cf. Definition 4.2)

2. $\forall x \exists y T_{2} x y$

3. $T_{i} \subseteq S_{i}$

4. $\forall x, y, z \in W\left(\left(S_{i} x y \wedge T_{i} y z\right) \Rightarrow T_{i} x z\right)$

5. $S_{E}=S_{1} \cup \ldots \cup S_{n}, T_{F}=T_{1} \cup \ldots \cup T_{n}$.

We denote the class of $K B$-models with $\mathcal{K B}$.

THEOREM 3.12

Each $K B$-consistent formula is satisfied in some $\mathcal{K B}$-model.

PROOF. If $\varphi$ is $K B$-consistent, it is contained in some $K B$-m.c. set $\Gamma$. So it is true in $\left(\mathcal{M}^{c}, \Gamma\right)$. We thus only have to show that $\mathcal{M}^{c}$ is a model in $\mathcal{K} \mathcal{B}$, i.e. that it satisfies $1-5$ of 3.11 .

1. $S_{2}^{c}$ is an equivalence: $S_{\imath}^{c}$ is reflexive, $S_{i}^{c} \Gamma \Gamma$, by definition of $S_{i}^{c}$ and, using $\mathrm{A} 2, K_{i} \varphi \in \Gamma \Rightarrow$ $\varphi \in \Gamma$. It is seen to be transitive, by an argument similar to that in the proof of item 4 of this theorem. Finally, it is symmetric: suppose $S_{i}^{c} \Gamma \Delta$, i.e. $K_{i} \varphi \in \Gamma \Rightarrow \varphi \in \Delta$ ( $^{*}$ ). If not $S_{i}^{c} \Delta \Gamma$, we have a $\psi$ with $K_{i} \psi \in \Delta$, but $\psi \notin \Gamma$. By item 2 a of 3.5 then, $\neg \psi \in \Gamma$, implying (using A2) $\neg K \psi \in \Gamma$. Axiom A3 guarantees $K_{i} \neg K_{i} \psi \in \Gamma$, so, by (*), $\neg K_{i} \psi \in \Delta$, which contradicts $K, \psi \in \Delta$.

2. By A9, $\left(\underline{B}_{i}\right.$ true $) \in \Gamma$, so, by 3.7 , for some $\Delta: T_{i} \Gamma \Delta$.

3. This was argued in 3.10 .

4. Suppose $S_{1}^{c} \Gamma \Delta$ and $T_{i}^{c} \Delta \Sigma$. Then, $B_{i} \varphi \in \Gamma \Rightarrow K_{i} B_{i} \varphi \in \Gamma$ (by A15), so (by definition of $T_{i}^{c}$ ), $B_{i} \varphi \in \Delta$ and hence (since $S_{i}^{c} \Delta \Sigma$ ) $\varphi \in \Sigma$. All in all, we have $T_{i} \Gamma \Sigma$. 
5. Since $\vdash E \varphi \rightarrow K_{i} \varphi$, as in 3.10 , we conclude $S_{i}^{c} \subseteq S_{E}^{c}$ for all $i \leq n$, and hence $S_{1}^{c} \cup \ldots \cup$ $S_{n}^{c} \subseteq S_{E}^{c}$. Now suppose $S_{E}^{c} \nsubseteq S_{1}^{c} \cup \ldots \cup S_{n}^{c}$, then for some $\Delta: S_{E}^{c} \Gamma \Delta$ and for no $i \leq n$, we have $S_{i}^{c} \Gamma \Delta$. Then, for all $i \leq n$, there is some $\varphi_{i}$ for which $K_{i} \varphi_{i} \in \Gamma$, but $\varphi_{i} \notin \Delta$. The former gives us $K_{i}\left(\varphi_{1} \vee \ldots \vee \varphi_{n}\right) \in \Gamma$ for all $i \leq n$ (and hence $E\left(\varphi_{1} \vee \ldots \vee \varphi_{n}\right) \in \Gamma$ ), and the latter $\left(\varphi_{1} \vee \ldots \vee \varphi_{n}\right) \notin \Delta$ (cf. 3.5.2b). This contradicts $S_{E}^{c} \Gamma \Delta$, so $S_{E}^{c} \subseteq S_{1}^{c} \cup \ldots \cup S_{n}^{c}$.

In [9], it is claimed that, if we would add the axioms for $C$ to the $S 5$-logic for knowledge, the necessity operator for $C$ may be seen as the transitive reflexive closure of $S_{E}$, i.e., $R_{C} u v$ iff there is some $S_{E}$-path from $u$ to $v$. From [7], where a similar operator $\left(\square^{*}\right)$ is studied in the area of dynamic logic, we know that the canonical model for such a system need not have this property. However, the canonical model is transferred into a finite model, which then is still a model of the proper kind and in which the relation that belongs to $\square^{*}$ is the reflexive transitive closure of the relation for $\square$. It may be shown that for $K B_{C D}$ there are similar problems, but also that a finite canonical model (of the appropriate kind) can be obtained in this case (cf. [17]). However, for the sequel, we need the unaffected canonical model as defined in 3.6.

Note how the particular properties of the binary relations in the canonical model are guaranteed by particular axioms of our logic. For instance, A2, $K_{i} \varphi \rightarrow \varphi$ forces $S_{i}$ to be reflexive, (3.12.1) and the definition of $E$ guarantees that $E$ may be understood as the necessity operator for the union of the operators $S_{i}$ for $K_{i}$. We emphasize that although $K_{z} \varphi \rightarrow \varphi$ is true on all $S_{i}$-reflexive models, the converse is not true: let $\mathcal{M}$ consist of two worlds $u$ and $v$, with $S_{\mathbf{z}}=\{(u, v),(v, u)\}$ and $\pi(u)=\pi(v)$. Then, $\mathcal{M}$ is not reflexive and still $\mathcal{M} \vDash K_{1} \varphi \rightarrow \varphi$, because of a particular property of a particular $\pi$. To abstract from the actual assignment $\pi$, the notion of frame is introduced, on which the interaction between axioms and properties on the binary relation can be studied more clearly.

DEFINITION 3.13

A frame $\mathcal{F}$ is a Kripke model without valuation

$$
\pi: \mathcal{F}=\left\langle W, S_{1}, \ldots, S_{n}, S_{E}, T_{1}, \ldots, T_{n}, T_{F}\right\rangle
$$

(in shorthand, $\mathcal{F}=\left\langle W, S_{i}, T_{i}\right\rangle$ ). We write $\mathcal{F} \vDash \varphi$ iff for all $\pi,\langle\mathcal{F}, \pi\rangle \models \varphi$. If $\Phi$ is any (first-order) property of $\mathcal{F}$, we say that multi modal formula $\varphi$ (which is generally understood to be a schema) corresponds with $\Phi$, if $\mathcal{F} \models \varphi \Leftrightarrow \mathcal{F}$ satisfies $\Phi$. We then write $\varphi \sim_{c o} \Phi$. If this is only true for frames $\mathcal{F}$ in some class $\mathcal{D}$ of frames, we say that we have relative correspondence $\left(\varphi \sim_{c o(D)} \Phi\right)$. For an introduction to this topic, we refer to [2]. We denote the class of models based on $\mathcal{F}$ by $\mathcal{M}(\mathcal{F})$. A given model $\mathcal{M}$ is understood to be based on its underlying frame $\mathcal{F}_{\mathcal{M}}$ : the underlying frame of the canonical model is called the canonical frame. Finally, we say that a logic $L$ is sound and complete with respect to $\mathcal{D}$, or $\left(L \vdash \varphi \Leftrightarrow \models_{\mathcal{D}} \varphi\right)$ if for all $\mathcal{F}$ in $\mathcal{D}, L$ $\vdash \varphi \Leftrightarrow \mathcal{F} \models \varphi$ (we then say that $\mathcal{F}$ is a frame for $L$ ).

\section{DEFINTION 3.14}

Let $\mathrm{M}_{\mathrm{L}}$ be some multi modal language for a normal modal logic $L$. We say that (the scheme) $\varphi \in M_{L}$ is canonical (can $(\varphi)$ ) if the canonical frame for $L$ satisfies $\varphi$.

As is known, (and as will be a consequence of the following section), on the level of frames, A2 does correspond to reflexivity. From 3.12.1 we know that $S_{\imath}^{c}$ in the canonical model for $K B$ is reflexive (forced by A2), and thus the canonical frame is. Since A2 $\sim_{c o}$ reflexivity, we conclude that $A 2$ is canonical. We stress that in general, the fact that an axiom A corresponds to property $\Phi$ is not equivalent to saying that $A$ is canonical ${ }^{2}$. We know that $A 5 \wedge A 6 \wedge A 7$ corresponds to

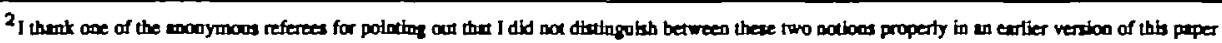




\section{Systems for Knowledge and Belief}

the property that $S_{C}$ (the relation for which $C$ is the necessity operator) is the reflexive transitive closure of $S_{E}$, whereas the canonical model for $K B$ need not have this property at all (cf. [7, 17]). Conversely, it may be that the canonical frame has some property $\Phi$ that is 'coincidental', i.e. that is not forced by any axiom. As an example, we saw that $(\mathrm{A} 2 \wedge \mathrm{A} 3)(\varphi)$ forces $S_{i}^{c}$ to be an equivalence relation. If $n=1$, since $\Gamma^{\prime}=\left\{K_{1} p, p\right\}$ and $\Delta^{\prime}=\left\{K_{1} \neg p, \neg p\right\}$ are both consistent sets, they give rise to worlds $\Gamma$ and $\Delta$ which are not $S^{c}$-accessible from each other. In other words, in the canonical frame $\exists x \exists y(\neg S x y \wedge \neg S y x)(\Phi)$ is true, although this property does not correspond to any modal formula (If $\mathcal{F}=\langle\{w\},\{(w, w)\}\rangle$, then $\mathcal{F} \vDash \varphi$, but $\mathcal{F} \not \models \Phi)$. It will appear that all the multi-modal schemes $\varphi$ in which we are interested here, are canonical.

REMARK 3.15

The fact that no modal formula $\varphi$ corresponds to a given $\neg \Phi$ is sometimes exploited to make a shift from a class of models $\mathcal{C}$ for which some logic $L$ is a complete axiomatization, to the class of models in $\mathcal{C}$ that do satisfy $\Phi$ (and for which $L$ is still a complete axiomatization)! For instance, on $S 5$-frames, $\neg \forall x \forall y(S x y \wedge S y x)$ does not (relatively) correspond to any $\varphi$; however, a move from the canonical model for $S 5$ to generated models gives models for which $\forall x \forall y(S x y \wedge S y x)$ holds (cf. [23,24]). Similarly, adding $\left(K_{1} \psi \vee \ldots \vee K_{n} \psi\right) \rightarrow D \psi$ (where the operator $D$ denotes 'distributed knowledge' cf. [9] or [14] in which this operator was represented with $I$ ) to our logic $K B$ would not give immediately a canonical model for which $S_{1} \cap \ldots \cap S_{n}=S_{D}$ holds (cf. [14]), where $S_{D}$ is the accessibility relation for which $D$ is the necessity operator. Now, the fact that $S_{1} \cap \ldots \cap S_{n} \neq S_{I}(=\neg \Phi)$ is not multi modally definable may be used to knead this canonical model into a model for which $\Phi$ is true, so that completeness of $K B \cup\left\{\left(K_{1} \psi \vee \ldots \vee K_{n} \psi\right) \rightarrow\right.$ $D \psi\}$ with respect to $\Phi$-models can be obtained (cf. [14]).

REMARK 3.16

A typical question we want to address using this machinery is the following. Suppose we have some epistemic logic $K B^{*}$ and we want to know whether adding one of our favourite properties for knowledge and belief implies having to accept another, perhaps less preferable property, i.e. we ask whether

$$
\text { (*) } K B^{*} \cup\left\{\varphi_{1}\right\} \vdash \varphi_{2} .
$$

The answer is positive, if, for example, we can show that $\operatorname{can}\left(\varphi_{1}\right)$, and find $\Phi_{1}$ and $\Phi_{2}$ such that $\varphi_{1} \sim_{c o} \Phi_{1}, \varphi_{2} \sim_{c o} \Phi_{2}$, and $\Phi_{1} \Rightarrow \Phi_{2}$. It is negative if we can find $\Phi_{h}$ with $\varphi_{h} \sim_{c o} \Phi_{h}(h=$ $1,2)$ and a $K B^{*}$-frame for $\Phi_{1}$ that does not satisfy $\Phi_{2}$, a question about first-order properties on Kripke frames. (Note that the seemingly semantical question whether the canonical model for $K B^{*} \cup\left\{\varphi_{1}\right\}$ is a model for $\varphi_{2}$ has a syntactical back bone: the answer is no iff $\neg \varphi_{2}$ is true at some world in $\mathcal{M}^{c}$ iff (by the fundamental theorem) $\neg \varphi_{2}$ is consistent in $K B^{*} \cup\left\{\varphi_{1}\right\}$.)

We end this section by mentioning an alternative semantics for our notions of knowledge and belief. In [8], Halpern shows that probabilistic Kripke models are also suitable to interpret S5like knowledge or $K D 45$-like belief. Such a model (for simplicity, we assume to have only one agent) $\mathcal{N}$ is of the form $\mathcal{N}=\langle W, P, \pi\rangle$, where $W$ is a finite or countably infinite set (of, again, worlds) and $\mathrm{P}: \mathcal{P}(W) \rightarrow[0,1]$ is a discrete probability function. In particular, $\square \varphi$ is true at $w$ iff $P(\{v \mid(\mathcal{N}, v) \vDash \varphi\})=1$ (cf. also $[12,22]$ ). It appears that, when no additional constraints are made upon $\mathrm{P}$, the logic for ' $\square$ ' is just $K D 45$, so that, in that case, 'belief' is the same as 'certainty'. If we want that 'certainty' is 'knowledge', we have to import the property $\square \varphi \rightarrow \varphi$, which is valid if we additionally assume that $P$ satisfies $\forall w P(w)>0$. In other words, $B \varphi \wedge \neg \varphi$ is satisfiable in world $w$, iff the measure of $w$ equals 0 (and $w$ is not taken into account when verifying $B \varphi$ at $w$ ). The techniques that we develop in the following section to characterize 
several properties for knowledge and belief, are easily extended to the models of this kind (which we make clear at the end of Section 4).

\section{Some correspondence results}

In this section, we will prove (among other properties) that axiom A15: $B_{i} \varphi \rightarrow K_{2} B_{i} \varphi$ corresponds with $\forall x \forall y \forall z\left(S_{i} x y \wedge T_{i} y z \rightarrow T_{i} x z\right)$. Given this, it is not difficult to see that T4: $K_{i} \varphi \rightarrow B_{i} K_{\imath} \varphi$ corresponds with $\forall x \forall y \forall z\left(T_{i} x y \wedge S_{\imath} y z \rightarrow S_{i} x z\right.$ ), (an interchange of the $K_{i}$ s and $B_{i} \mathrm{~s}$ induces an interchange of the $S_{i} \mathrm{~s}$ and $\left.T_{2} \mathrm{~s}\right)$ and also that $\left(K_{2} \varphi \rightarrow K_{2} K_{2} \varphi\right)$ corresponds with $\forall x \forall y \forall z\left(S_{i} x y \wedge S_{i} y z \rightarrow S_{i} x z\right)$, transitivity of $S_{i}$ (replacing $B_{i}$ by $K_{i}$ induces a replacement of $T_{\imath}$ by $S_{\imath}$ ). Obviously, inferring the last mentioned correspondence from one of the first two is easy, whereas the other way around is a much more difficult, if not impossible, task. So, for correspondence-problems, it would be nice having different operators for each occurrence in formulas like A15.

DEFINITION 4.1

We assume to have a language with sufficiently many operators $K^{1}, K^{2}, K^{3}, \ldots$ and equally many binary relations $R^{1}, R^{2}, R^{3}, \ldots$ associated to them. The $K^{m}$ 's $(m \in \mathbb{N})$ are just modal operators, which could be instantiated with operators from $\left\{K_{i}, B_{i} \mid\right.$ $i \leq n\}$.

DEFINITION 4.2

We define the following properties on binary relations $R^{1}, R^{2}$ and $R^{3}$, leaving universal quantification over $x, y$ and $z$ implicit.
(a) $\quad$ seriality of $R^{1}$
(b) $\quad$ reflexivity of $R^{1}$
(c) transitivity of $R^{1}$ over $\left(R^{2}, R^{3}\right)$
(d) Euclidicity of $R^{3}$ over $\left(R^{2}, R^{1}\right)$
(e) weak $\left(R^{1}, R^{2}\right)$-density of $R^{3}$
(f) selective transitivity of $R^{1}$ over $\left(R^{2}, R^{3}\right)$
(g) $\quad R^{1}$-postponed reflexivity of $R^{2}$
(h) $\quad R^{2}$-symmetry of $R^{1}$

$$
\begin{array}{r}
\exists y R^{1} x y \\
R^{1} x x \\
R^{2} x y \wedge R^{3} y z \Rightarrow R^{1} x z \\
R^{2} x y \wedge R^{1} x z \Rightarrow R^{3} y z \\
R^{3} x y \Rightarrow\left(\exists z\left(R^{1} x z \wedge R^{2} z y\right)\right. \\
\exists y \forall z\left(R^{2} x y \wedge\left(R^{3} y z \Rightarrow R^{1} x z\right)\right) \\
R^{1} x y \Rightarrow R^{2} y y \\
R^{1} x y \Rightarrow R^{2} y x
\end{array}
$$

If, for instance, we have transitivity of $R$ over $(R, R)$, we say that $R$ is transitive. An equivalence relation is reflexive, transitive and symmetric.

THEOREM 4.3

Consider the following multi modal formulas (in $K^{1}, K^{2}$ and $K^{3}$ ).
(a) $\neg K^{1}$ false
(b) $K^{1} \varphi \rightarrow \varphi$
(c) $K^{1} \varphi \rightarrow K^{2} K^{3} \varphi$
(d) $\neg K^{1} \varphi \rightarrow K^{2} \neg K^{3} \varphi$
(e) $K^{1} K^{2} \varphi \rightarrow K^{3} \varphi$
(f) $K^{1} \neg K^{2} \varphi \rightarrow \neg K^{3} \varphi$
(g) $K^{1}\left(K^{2} \varphi \rightarrow \varphi\right)$
(h) $\varphi \rightarrow K^{1} \neg K^{2} \neg \varphi$

Then, for all $x \in\{a, \ldots, h\}$ :

1. as a scheme, 4.3.x corresponds with 4.2.x

2. axiom 4.3.x is canonical. 
PROOF. A proof for 1 is obtained by generalizing well known correspondence results for (standard) modal logic (cf. $[2,18]$ ). In fact, both 1 and 2 follow from a theorem ascribed to Sahlqvist, but proven independently in [26] and [1] (cf. also [25]). Here we do not need that full machinery, but prove 1(d), as an example. For item 2, one needs generalizations of the construction in Section 3, from which the results for (a), (b), (c) and (g) are immediately obtained. To illustrate an existential quantified case, we prove 2(e) as a generalisation of a proof in [7].

1(d). We have to show:

$$
\mathcal{F} \models \neg K^{1} \varphi \rightarrow K^{2} \neg K^{3} \varphi \Leftrightarrow \mathcal{F} \models\left(R^{2} x y \wedge R^{1} x z \Rightarrow R^{3} y z\right) .
$$

$\Leftarrow$ : Suppose for some $\pi$ and $w,\langle\mathcal{F}, \pi\rangle, w \vDash \neg K^{1} \varphi$, i.e., for some $v$ with $R^{1} w v$, $\langle\mathcal{F}, \pi\rangle, v \models \neg \varphi$. Let $u$ be any world for which $R^{2} w u$. Then $R^{3} u v$, and hence $\langle\mathcal{F}, \pi\rangle, u \models$ $\neg K^{3} \varphi$, and thus $\langle\mathcal{F}, \pi\rangle, w \models K^{2} \neg K^{3} \varphi$.

$\Rightarrow$ : Suppose $\mathcal{F} \not R^{2} x y \wedge R^{1} x z \Rightarrow R^{3} y z$, i.e., there are worlds $u$, $v$, and $w$ for which $R^{2} w u, R^{1} w v$, but not $R^{3} u v$. Define $\pi$ such that $p$ is false only in $v$. Then $\langle\mathcal{F}, \pi\rangle, w \models$ $\neg K^{1} p \wedge \neg K^{2} \neg K^{3} p$, so $\mathcal{F} \not \forall \neg K^{1} p \rightarrow K^{2} \neg K^{3} p$.

2(e). Suppose $R^{3} \Gamma \Delta$. We have to find a $\Sigma$ in the canonical model, for which both $R^{1} \Gamma \Sigma$ and $R^{2} \Sigma \Delta$. By the definition of canonical model, and Lemma 3.5.1, it is sufficient to show that the set $\Sigma^{\prime}=\left\{\psi \mid K^{1} \psi \in \Gamma\right\} \cup\left\{K^{2} \delta \mid \delta \in \Delta\right\}$ is consistent. Suppose not, then $\psi_{1} \wedge \ldots \wedge$ $\psi_{m} \wedge \underline{K^{2}} \delta_{1} \wedge \ldots \wedge \underline{K^{2}} \delta_{k} \rightarrow \perp$, for some $m, k \geq 1$. This is equivalent to $\psi_{1} \wedge \ldots \wedge \psi_{m} \rightarrow$ $\left(K^{2} \neg \delta_{1} \vee \ldots \vee K^{2} \neg \delta_{k}\right)$, so, using 2.5 (iii), we have $\psi_{1} \wedge \ldots \wedge \psi_{m} \rightarrow K^{2}\left(\neg \delta_{1} \vee \ldots \vee \neg \delta_{k}\right)$. By 2.5(i) and (ii), we get $K^{1} \psi_{1} \wedge \ldots \wedge K^{1} \psi_{m} \rightarrow K^{1} K^{2}\left(\neg \delta_{1} \vee \ldots \vee \neg \delta_{k}\right)$. We now use $K^{1} K^{2} \varphi \rightarrow K^{3} \varphi: K^{1} \psi_{1} \wedge \ldots \wedge K^{1} \psi_{m} \rightarrow K^{3}\left(\neg \delta_{1} \vee \ldots \vee \neg \delta_{k}\right)$. By definition of the $\psi_{r}, K^{1} \psi_{r} \in \Gamma(r \leq m)$. Since $R^{3} \Gamma \Delta$, we have $\left(\neg \delta_{1} \vee \ldots \vee \neg \delta_{k}\right) \in \Delta$, and (using 2c) $\neg \delta_{s} \in \Delta$, for some $s \leq k$, contradicting the definition of the $\delta$ s.

We like to stress that the proofs for these general cases ('fresh' operators for each occurrence) are not more complicated than in the standard modal case.

\section{REMARK 4.4}

Because $K^{2} \varphi \rightarrow \varphi$ corresponds with reflexivity of $R^{2},\left(\forall x R^{2} x x\right)$, it is easy to see that $K^{1}\left(K^{2} \varphi \rightarrow \varphi\right)$ is valid at $x$ if all $R^{1}$-successors $y$ of $x$ satisfy $K^{2} \varphi \rightarrow \varphi$, and so, if $\forall y\left(R^{1} x y \rightarrow R^{2} y y\right)$. This suggests a way to derive 'postponed correspondences' like $4.3(\mathrm{~g})$. Suppose $K B^{m}$-formula $\varphi$ corresponds locally with property $\phi(x)$, i.e. for all frames $\mathcal{F}$ and world $x \in \mathcal{F},(\mathcal{F}, x) \models \varphi$ iff $(\mathcal{F}, x) \models \phi(x)$. Then, $K^{i} \varphi$ corresponds locally with $\forall y\left(R^{i} x y \rightarrow \phi(y)\right)$ (and so, globally with $\forall x \forall y\left(R^{i} x y \rightarrow \phi(y)\right)$.

One can now systematically list all the properties that the relations $S_{i}$ and $T_{i}$ of the frames in $\mathcal{K B}$ satisfy, by investigating the axioms involving $K_{i}$ and $B_{i}$. For instance, for transitivity, we get, that from the c-part of 4.3.1, it follows that $S_{i}$ and $T_{z}$ are transitive (from Lemma 2.2 and T6). $T_{i}$ is transitive over $\left(S_{i}, T_{i}\right)$ (A15), $S_{i}$ is 'maximally transitive': it is transitive over $\left(S_{1}, S_{i}\right)$, over $\left(T_{i}, S_{2}\right)$ (T4), over $\left(S_{i}, T_{i}\right)$ (because $K_{i} \varphi \Rightarrow_{A 2} B_{i} \varphi \Rightarrow_{A 15} K_{i} B_{i} \varphi:$ T11) and over $\left(T_{i}, T_{i}\right)\left(K_{i} \varphi \Rightarrow_{T 11} K_{i} B_{i} \varphi \Rightarrow_{A 14} B_{i} B_{i} \varphi: T 12\right)$. We can now do some reasoning about properties of binary relations in $\mathcal{K}$. and translate the result to $K B$.

\section{THEOREM 4.5}

1. A reflexive, Euclidean relation is both symmetric and transitive.

2. If $R^{1}$ is Euclidean and reflexive, $R^{2} \subseteq R^{1}$ and $R^{2}$ is transitive over $\left(R^{1}, R^{2}\right)$, then $R^{2}$ is Euclidean.

3. A relation that is Euclidean, is also postponed reflexive. 
ProOF. 1. Suppose $R x y$ (1) and $R y z$ (2). By reflexivity, $R x x$ (3). Euclidicity, (1) and (3) give $R y x$ (4). This proves symmetry of $R$. Finally, (2), (4) and Euclidicity give $R x z$.

2. Suppose $R^{2} x y$ and $R^{2} x z$. Then $\left(R^{2} \subseteq R^{1}\right) R^{1} x y$ and (by ii) $R^{1} y x$. Since $R^{2}$ is transitive over $\left(R^{1}, R^{2}\right)$, and $R^{1} y x$ and $R^{2} x z$, we have $R^{2} y z$.

3. Rxy and Euclidicity give Ryy.

Using arguments of 3.16, we find $K B \vdash \varphi \rightarrow K_{1} \neg K_{i} \neg \varphi$, combining results on correspondences and canonicalness in the following way: we have $K B \vdash K_{i} \varphi \rightarrow \varphi$ and $K B \vdash \neg K_{i} \varphi \rightarrow K_{i} \neg K_{i} \varphi$ (A2 and A3); 4.3.2(b and d) guarantee that the canonical frame for $K B$ also validates $\mathrm{A} 2$ and $\mathrm{A} 3$. Now we use 4.3.1(b and d) to conclude that $S_{\mathfrak{z}}$ on this frame is both reflexive and Euclidean, and thus, by 4.5.1, symmetric. By 4.3.1(h), we know that the canonical frame (and hence, also the canonical model) for $K B$ satisfies $\varphi \rightarrow K_{i} \neg K_{i} \neg \varphi$; so, using the fundamental theorem (3.8) we observe that $\varphi \rightarrow K_{i} \neg K_{i} \neg \varphi$ is contained in every $K B$-maximal consistent set and hence, by 3.5.2(d), $K B \vdash \varphi \rightarrow K_{2} \neg K_{i} \neg \varphi$.

Note that, in a similar way, we conclude that $S_{1}$ is transitive, so that we again have a proof of positive introspection for $K_{3}$. Whereas in 2.2, we argued that A2 and A3 were sufficient to derive the same result within $K B$, we now semantically use $\Phi_{2}$ and $\Phi_{3}$ with $\mathrm{A} 2 \sim_{c o} \Phi_{2}$ and A3 $\sim_{c o} \Phi_{3}$ to find a $\Phi$ with $\left(\Phi_{2} \wedge \Phi_{3}\right) \Rightarrow \Phi$ and $\Phi \sim_{c o} K_{i} \varphi \rightarrow K_{i} K_{i} \varphi$. There is a similar correspondence between the proof of negative belief introspection in 2.2. and deriving Euclidicity for $T_{i}$ from 4.5.2. Finally, note that 4.5.3 gives us T8 again: since $\forall x \forall y\left(T_{i} x y \rightarrow T_{i} y y\right)$ is derived for $T_{i}$, using 4.4 we conclude that $B_{i}\left(B_{i} \varphi \rightarrow \varphi\right)$ is derivable in $K B$; which we indeed showed in 2.8 .

In particular, note that the $T_{i} \mathrm{~s}$ in the frames of $\mathcal{K B}$ are also transitive, Euclidean and dense (this follows from 4.3 together with $\mathrm{T} 6\left({ }^{\prime} \rightarrow\right.$ '), $\mathrm{T} 7\left({ }^{\prime} \rightarrow\right.$ ') and $\mathrm{T} 6($ ' $\leftarrow$ ') of 2.6 , respectively). In the opposite direction, one can make an exhaustive list of properties of 4.2 for the frames in $\mathcal{K} B$ (which immediately proves the following theorem), and use the absence of special properties in $\mathcal{K B}$ to show non-derivability in $K B$. For example, $T_{i}$ is not transitive over $\left(T_{i}, S_{2}\right)$ and also not over $\left(S_{i}, S_{i}\right)$. By way of example, we prove the former. Figure 1 is a $\mathcal{K} \mathcal{B}$-structure, in which $T_{i}$ is denoted with thick, and $S_{i}$ with thin arrows, respectively. Note that although $T_{i} u v$ and $S_{\imath} v w$ are true in that structure, $T_{i} u w$ is not.

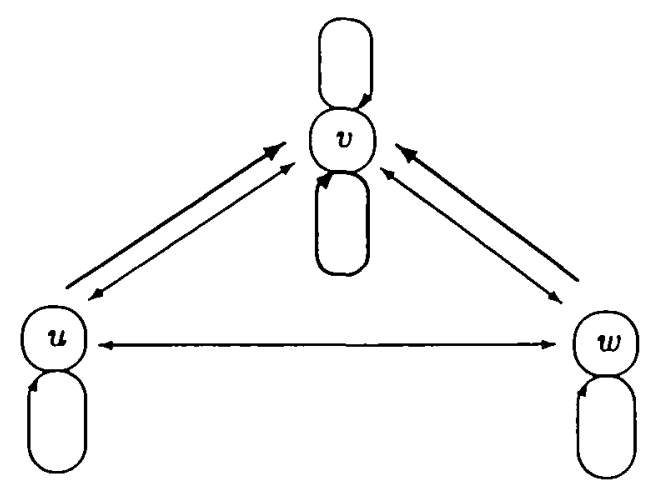

FlG. 1. A $\mathcal{K B}$-structure, in which $S_{z}$ is denoted with thin, and $T_{i}$ with thick arrows, respectively 
From the previous paragraph, we get a lot of non-theorems of $K B$ : in particular, because $T_{2}$ is not transitive over $\left(T_{i}, S_{i}\right)$, we have $K B \not \forall B_{i} \varphi \rightarrow B_{i} K_{i} \varphi$. We give a list (writing ' $\forall \varphi^{\prime}$ ' instead of ' $K B \forall \varphi^{\prime}$ ') of non-derivable formulas that are important when studying knowledge and belief (cf. the introduction, 4.7, and, for a classification, Section 6).

\section{THEOREM 4.6}

1. $\forall B_{1} \varphi \rightarrow B_{i} K_{i} \varphi, \forall B_{2} \varphi \rightarrow K_{i} K_{i} \varphi$

2. $\forall \neg K_{i} \varphi \rightarrow K_{i} \neg B_{i} \varphi, \forall \neg \neg K_{i} \varphi \rightarrow B_{i} \neg B_{i} \varphi$

3. $\forall B_{i} B_{i} \varphi \rightarrow K_{i} \varphi, \not \forall K_{i} B_{i} \varphi \rightarrow K_{i} \varphi$

4. $\forall B_{i} \neg K_{i} \varphi \rightarrow \neg B_{i} \varphi, \forall K_{i} \neg K_{i} \varphi \rightarrow \neg B_{i} \varphi$

5. $\forall K_{i}\left(B_{i} \varphi \rightarrow \varphi\right)$

6. $\left.\left.\forall \varphi \rightarrow K_{i}\right\urcorner B_{i} \neg \varphi, \forall \varphi \rightarrow B_{i} \neg B_{i}\right\urcorner \varphi$.

\section{REMARK 4.7}

Of Theorem 4.6, 1 and 3 are of the form $X B \varphi \rightarrow Y K \varphi$, with $X, Y \in\{B, K, \epsilon\}$, where $\epsilon$ is the empty (identity) operator. Properties 2 and 4 express that if $\varphi$ is (believed or known to be) not known, it should also have consequences for the agent's (non-) belief about $\varphi$ (they are of the form $X \neg K \varphi \rightarrow Y \neg B \varphi, X, Y \in\{B, K, \epsilon\}$ ). So, in $K B$, it is perfectly well possible (i.e. satisfiable in the system $K B$ ), that an agent (knows or believes that he) believes $\varphi$, without (knowing or believing that he is) knowing $\varphi$. The non-theorems of 4.6 neatly show some differences between knowledge and belief: $1-5$ of 4.6 are all valid in $K B$ if we replace each occurrence of $B$ by $K$.

We end this section with the following aside. The correspondences that are obtained here, can directly be transformed to the general probability structures (g.p.s.) as introduced in [8] (cf. the end of Section 3). To see this, we first generalize the notion of g.p.s. A structure $\mathcal{N}$ is a g.p.s.k if $\mathcal{N}=\left\langle W, \mathcal{P}_{1}, \ldots \mathcal{P}_{k}, \pi\right\rangle$, with $W$ a (finite or countable) set of worlds, $\pi$ a truth-assignment for each world and the $\mathcal{P}_{i}$ 's families of discrete probability functions (a function $P_{i}(w)$ for each world $w$ ) on $W(i \leq k)$. Following [8], we define the support $t_{i}$ relation on $W$ as $(u, v)$ in support $t_{2}$ iff $\mathcal{P}_{i}(u)(v)>0$. Under this definition, we can view a g.p.s. $k$ as a Kripke structure with $k$ accessibility relations (the support relations). It is obvious that any result on modal logic has immediate implications for probability structures via this support relation. For instance, $K^{1} \varphi \rightarrow K^{2} K^{3} \varphi$ will be valid on those structures for which $\forall x y z\left(P_{2}(x) y>0 \wedge P_{3}(y) z>0 \Rightarrow P_{1}(x) z>0\right)$ holds.

\section{Conscious beliefs, believed consciousness}

Our system $K B$ verifies A15: $B_{i} \varphi \rightarrow K_{i} B_{i} \varphi$ (beliefs are 'conscious', in the sense of 'known'). This demonstrates that $B_{i}$ represents a rather explicit belief, in the sense that the agent is aware of adopting them-the terms 'explicit belief' and 'implicit belief' are introduced in [22] and also used in $[20]$; in $[5,15]$ these notions are related to 'awareness'. Here, one may just associate 'implicit' with 'weak' and 'explicit' with 'strong'. Knowledge might be considered a very explicit notion of belief. If $B_{i}$ would represent a notion of implicit belief, it seems reasonable to let $\left(B_{\imath} \varphi \wedge B_{i} \neg \varphi\right.$ ) be satisfiable simultaneously with $\neg B_{2} \perp$ (cf [15]; however, at this point, our use of 'implicit belief' diverges from that in $[5,20,22]$, where it is assumed to be some logically closed set of beliefs-facts that implicitly follow from the agent's beliefs, although he need not be aware of it). But assuming (satisfiability of) $\left(B_{i} \varphi \wedge B_{i} \neg \varphi\right)$, A 15 would yield $K_{i}\left(B_{i} \varphi \wedge B_{i} \neg \varphi\right)$. This again demonstrates that $A 15$ is reasonable for explicit beliefs (in our sense); if agent $i$ knows that he has inconsistent beliefs, he should retract some of them. 
Kraus and Lehmann remark that it would be interesting to also have $B_{i} \varphi \rightarrow B_{i} K_{i} \varphi$, implying that agent $i$ believes that his beliefs are conscious. (In Section 6, we pay some more attention to the kinds of belief these two formulas would apply to.) However, adding $B_{\imath} \varphi \rightarrow B_{i} K_{i} \varphi$ to $K B$ would give $B_{i} \varphi \rightarrow K_{i} \varphi$. Now, we concentrate on finding $K B$-like systems that do allow $B_{\mathfrak{i}} \varphi \rightarrow B_{\mathfrak{i}} K_{\mathfrak{i}} \varphi$, without yielding $\left(B_{i} \varphi \leftrightarrow K_{\mathfrak{i}} \varphi\right)$. (We will say that such a system solves the $\mathrm{B}$ (elieved) $\mathrm{C}$ (onsciousness) of $\mathrm{B}$ (eliefs) problem.) The latter property $\left(\vdash K_{i} \varphi \leftrightarrow B_{i} \varphi\right)$ corresponds with $S_{\imath}=T_{2}$, for which we will give a sufficient condition. Recall from Theorem 4.3 that $B_{i} \varphi \rightarrow B_{i} K_{i} \varphi$ corresponds with $\forall s \forall t \forall u: T_{i} s u \wedge S_{i} u t \Rightarrow T_{i} s t$

\section{THEOREM 5.1}

Let $S$ and $T$ be two binary relations on a set $W$, and consider the properties:

(a) $T$ is transitive over $(T, S)$

(b) $T$ is contained in $S$

(c) $T$ is serial, and

(d) $S$ is Euclidean

Then:

1. ( $S$ and $T$ satisfy (a) $-(\mathrm{d})) \Rightarrow(S$ equals $T)$

2. For each proper subset $A \subset\{a, b, c, d\}$, we can find relations $S$ and $T$ that satisfy $A$, but for which $S \neq T$.

ProOF. We prove 1, and refer to Fig. 2 for an example of a structure that satisfies $a, b$ and $c$, but for which $S \neq T$. So suppose $S x y$. Using $c$, we find a $z$ for which $T x z$ and, by $b, S x z$. By $d$, we get $S z y$. Now apply $a$ to $T x z$ and $S z y$ to conclude $T x y$.

Semantically, we now know when $S$ and $T$ do collapse. What does this mean for knowledge and belief? From 4.3 we know that $B_{i} \varphi \rightarrow B_{i} K_{i} \varphi(1)$ characterizes 5.1(a), that $K_{i} \varphi \rightarrow B_{i} \varphi$ (2) characterizes 5.1 (b), that $\neg B_{i}$ false (3) characterizes 5.1 (c) and that $\neg K_{i} \varphi \rightarrow K_{1} \neg K_{i} \varphi(\underline{4})$ characterizes 5.1(d). Now it is clear, that, if we want $B_{i} \varphi \rightarrow B_{i} K_{i} \varphi$ but not $B_{i} \varphi \rightarrow K_{i} \varphi$, we have to give up one of the three last properties of $\underline{2}, \underline{3}$, and $\underline{4}$ for knowledge and belief, because of the following:

THEOREM 5.2

$\vDash(\underline{1} \wedge \underline{2} \wedge \underline{3} \wedge \underline{4}) \rightarrow\left(B_{i} \varphi \rightarrow K_{i} \varphi\right)$

ProOf. Apply 3.16 to 5.1.

Theorem 5.2 implies that adding $B_{i} \varphi \rightarrow B_{i} K_{i} \varphi$ to $K B$ does yield $B_{i} \varphi \equiv K_{i} \varphi$, because $K B$ satisfies $\underline{2}, \underline{3}$ and $\underline{4}$. Together with 5.1 it also offers solutions: if one wants to have $B_{i} \varphi \rightarrow B_{i} K_{i} \varphi$ but not $B_{i} \varphi \rightarrow K_{\imath} \varphi$, one has to give up one of the properties expressed by A14, A9 or A3. Summarizing, Theorem 5.1.1 implies that, in order to add $\left(B_{i} \varphi \rightarrow B_{i} K_{i} \varphi\right)$ and at the same time avoiding $\left(B_{i} \varphi \equiv K_{i} \varphi\right)$, it is necessary to give up one of A3, A9 and A14, whereas 5.1.2 expresses that this may also be sufficient (whether this is indeed so, depends on the axioms we do add to such a system; in the sequel, we will investigate several possibilities).

Giving up $A 14, K_{i} \varphi \rightarrow B_{i} \varphi$, or semantically, $T_{i} \subseteq S_{i}$, makes $\left(K_{i} p \wedge \neg B_{i} p\right)$, and even $\left(K_{i} p \wedge B_{i} \neg p\right)$ satisfiable. Then, $B_{i}$ represents an implicit notion of belief-a notion that we studied in [15]-and then the whole system $\mathrm{KB}$ needs revision. (See also [28] for an epistemic logic in which $\left(K_{i} \varphi \rightarrow B_{i} \varphi\right)$ is not valid.) We doubt whether, for instance, $A 15$ would be 


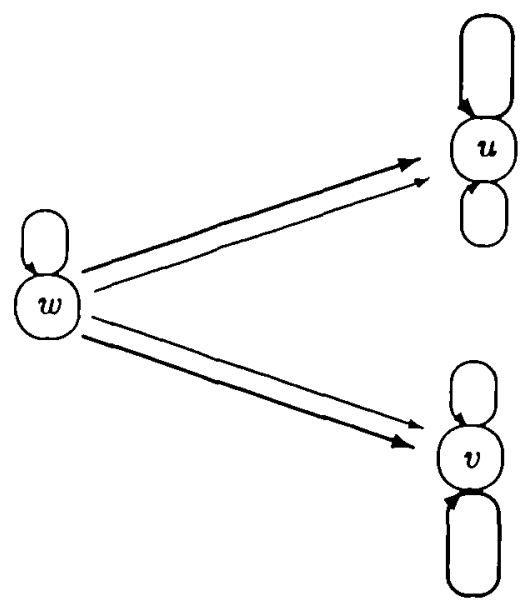

FIG. 2. A structure in which $S$ is denoted with thin, and $T$ with thick arrows

a desirable property for implicit belief, and probably the same holds for $B_{\imath} \varphi \rightarrow B_{\imath} K_{i} \varphi$, the formula that urged us to drop A14 in the first place.

One could also give up A9, but similar remarks as in the previous paragraph can be made here. For instance, from A15, we get $B_{i}$ false $\rightarrow K_{i} B_{i}$ false, but why should an agent hold on to false beliefs if he knows he has them? Moreover, dropping $\neg B_{i}$ false cannot invalidate $\neg B_{i}$ false $\rightarrow\left(K_{i} \varphi \leftrightarrow B_{i} \varphi\right)$. So, either agent $i$ beliefs in falsehood, or his beliefs equal his knowledge. Dropping $\mathrm{A} 3, \neg K_{i} \varphi \rightarrow K_{i} \neg K_{i} \varphi$ is the third alternative. Note that a knowledge agent that satisfies A3 is very much aware of all the facts that are around: if he does not know $\varphi$, he knows that he does not. This would imply, that a Bantu tribesman knows that he does not know that personal computer prices are going down. For a discussion about 'awareness', we refer to [5], where the Bantu tribesman example is taken from, and to [15].

From Remark 2.2, we know that $\neg K_{i} \varphi \rightarrow K_{2} \neg K_{i} \varphi$ (A3) implies $K_{i} \varphi \rightarrow K_{i} K_{i} \varphi\left(\mathrm{A}^{\prime}\right)$. We could try to see what happens if we replace $\mathrm{A} 3$ by $\mathrm{A}^{\prime}$ (a discussion on these axioms can already be found in [10]). We know that $\mathrm{A}^{\prime}$ corresponds with transitivity of $S_{\imath}$.

\section{DEFINITION 5.3}

Let $K B^{-}$be the system consisting of all the axioms of $K B$, but with $\mathrm{A} 3$ replaced by $\mathrm{A} 3^{\prime}$ : $K_{1} \varphi \rightarrow K_{i} K_{i} \varphi$ and with $\mathrm{A} 17: B_{\mathfrak{i}} \varphi \rightarrow B_{\mathrm{i}} K_{i} \varphi$, added to it.

\section{THEOREM 5.4}

$K B^{-} \forall B_{i} \varphi \rightarrow K_{i} \varphi$.

PROOF. To prove this, from arguments given in this section, it is clear that it is sufficient to find a $K \mathcal{B}^{-}$model $\mathcal{M}$ in which the $S_{i} \mathrm{~s}$ are reflexive and transitive, the $T_{2} \mathrm{~s}$ are serial and transitive (not Euclidean; note that 4.5.2 cannot be applied in $\mathcal{K B ^ { - }}$ ), $T_{i} \subseteq S_{i}$ and in which the $T_{i}$ s are transitive over both $\left(S_{i}, T_{i}\right)$ and $\left(T_{2}, S_{i}\right)$, but at the same time $S_{i} \notin T_{i}$. Such a structure is given in Fig. 2.

From the model of Fig. 2, we see that, since $T_{i}$ is not Euclidean over $\left(S_{i}, T_{i}\right)$, we also have $K B^{-} \forall \neg B_{i} \varphi \rightarrow K_{\mathbf{i}} \neg B_{i} \varphi$. We will investigate the (non-) theorems of ' $K B$-like systems' a 
bit more systematically in the next section. Of course, it is easy to define a system that does not verify $B_{i} \varphi \rightarrow K_{i} \varphi$ but that does yield $\mathrm{A} 15$ and $\mathrm{A} 17$. However, we want a system $S$ that is 'close(st) to $K B \cup\{A 17\}$ ' and such that $S \forall B_{i} \varphi \leftrightarrow K_{i} \varphi$. For such an $S$, some theorems of $K B$ must be sacrificed. For example, $B_{i} K_{i} \varphi \rightarrow K_{i} \varphi$ (implied by T4), with $B_{i} \varphi \rightarrow B_{i} K_{i} \varphi$ immediately yields $B_{1} \varphi \rightarrow K_{i} \varphi$. In order to study these problems more systematically and to get a clearer notion of 'close to $K B$ ' we will explore the fact that we now know how the properties of knowledge and belief, as expressed in the axioms and theorems T1-T10 of $K B$ act upon the structure of its Kripke models.

\section{Introspection and extraspection}

Now, before we take up the BCB-problem itself, we will investigate some general properties of knowledge and belief. We will see how they are present in $K B$, and we show some combinations of those properties that are possible in a system that defines knowledge and belief as two necessity operators.

\section{DEFINITION 6.1}

Let $X, Y$ and $Z$ range over epistemic operators. Then, formulas of the form:

(a) $X \varphi \rightarrow Y Z \varphi$ are called positive introspection (p.i.-) formulas

(b) $\neg X \varphi \rightarrow Y \neg Z \varphi$ are called negative introspection (n.i.-) formulas

(c) $X Y \varphi \rightarrow Z \varphi$ are called positive extraspection (p.e.-) formulas

(d) $X \neg Y \varphi \rightarrow \neg Z \varphi$ are called negative extraspection (n.e.-) formulas

(e) $X(Y \varphi \rightarrow \varphi)$ are called trust formulas.

We will call instantiations of (a)-(d) inspection-formulas, and we will denote the set of all instantiations of (a)-(e) with $I T$. Each of the above defined notions (a)-(e) determines a subclass of $I T$.

Note that all the axioms and theorems that were discussed or given in Section 2 were equivalent to either an $I T$-formula, or of one of the forms $X \varphi \rightarrow Y \varphi$ and $X \varphi \rightarrow \varphi$.

THEOREM 6.2

In any system, if $\left(K_{i} \varphi \rightarrow B_{i} \varphi\right)$ (A14) is valid, each class of $I T$ is partially ordered, with $\varphi \leq \psi$ iff $\varphi \Rightarrow{ }_{A 14} \psi$. For each class of $I T$, there is a smallest element (modulo equivalence).

PROOF. We define the notions of positive and negative occurrences of operators $X$ in formulas. If $\varphi$ does not contain $X, X$ occurs positively in $X \varphi$. Each positive (negative) occurrence of $X$ in $\varphi$ is a positive (negative) occurrence of $X$ in $Y \varphi$ ( $Y$ may be $X, \epsilon$, or any other modal operator) and $\psi \rightarrow \varphi$. Each positive (negative) occurrence of $X$ in $\varphi$ is a negative (positive) occurrence of $X$ in $\neg \varphi$ and $\varphi \rightarrow \psi$. Now we can show that $\varphi \geq \psi$ iff $\psi$ can be obtained from $\varphi$ by replacing negative occurrences of $B_{i}$ in $\varphi$ by $B_{i}$ or $K_{i}$, and replacing positive occurrences of $K_{i}$ by $B_{i}$ or $K_{i}$. Instead of a proof, we give an example: in Fig. 3, ' $\geq$ ' is the transitive reflexive closure or the relation denoted with arrows in the class $N I$ (we do not write the subscript i; formulas in rectangles are non-theorems of $K B$ ).

In the next paragraphs, we will spend some words on positive introspection, followed by a paragraph about negative introspection. The discussion can easily be extended to the other inspection properties. To start, we want to point out the difference between $(X \varphi \rightarrow Y Z \varphi)$ and $Y(X \varphi \rightarrow Z \varphi)$. Note that the latter is purely a property of $Y$-beliefs, whereas one could interpret the former as a property noted by an observer from outside. Compare the difference between $\left(K_{\imath} \varphi \rightarrow K_{j} K_{k} \varphi\right)$ and $K_{\jmath}\left(K_{i} \varphi \rightarrow K_{k} \varphi\right)$ : in the latter formula, the fact that agent $k$ 


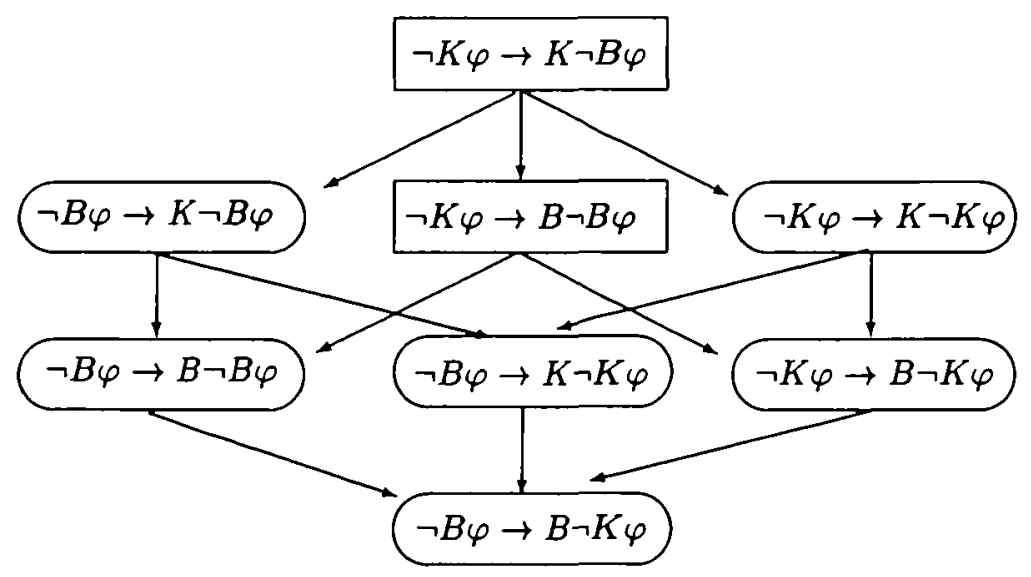

FIG. 3. ' $\geq$ ' in the class $N I$

knows everything that $i$ knows, is known by agent $j$ (i.e. in the scope of $K$,!). Even clearer is the distinction between $(B \varphi \rightarrow K B \varphi)$ and $K(B \varphi \rightarrow B \varphi)$.

The positive introspection formula $(X \varphi \rightarrow Y Z \varphi)$ has, if $(K \varphi \rightarrow B \varphi)$ is valid, as a strongest instantiation $(B \varphi \rightarrow K K \varphi)$, and as its weakest $(K \varphi \rightarrow B B \varphi)$. For 'ordinary' belief and knowledge, the first is indeed too strong. The latter presumes introspection in one's own beliefs. We doubt, however, whether people use phrases like 'I believe that I believe that ...' ', and if so, they probably mean 'I very weakly believe that ...'. It would be interesting to have a system with two (possibly the same) notions of belief, say explicit belief $\left(B^{e}\right)$ and implicit belief $\left(B^{i}\right)$, such that $\left(B^{2} B^{e} \varphi \wedge \neg B^{e} \varphi\right)$ is satisfiable.

Because $K \varphi \rightarrow K K \varphi$ is true for most notions of knowledge, it seems reasonable to expect that $(B \varphi \rightarrow B K \varphi)$ is true for notions of belief that resemble knowledge, i.e. for strong notions of belief. We can be a bit more precise here, and ask for which $X$ and $Y,(X \varphi \rightarrow Y K \varphi)$ should be true. We might expect $(X \varphi \rightarrow Y K \varphi)$ to hold for 'strong' $X$-belief, and 'weak' $Y$-belief. For instance, the choices $X \equiv$ 'I am convinced' and $Y \equiv$ 'I suspect' is a more acceptable than the other way around. (In $K B,(K \varphi \rightarrow B K \varphi)$ is valid, whereas $(B \varphi \rightarrow K K \varphi)$ is not.)

Instead of 'strong' belief, we could also write 'expensive' (having serious consequences, e.g. the belief of a judge or surgeon), and instead of 'weak' we could say 'cheap' (e.g. the belief of some gossip-paper). (The introspection property $B \varphi \rightarrow K B \varphi$ seems desirable when $B$ denotes an 'expensive' belief; for instance, if a judge believes that females are bad car-drivers, he had better know that he believes so when he has to judge about Alice's role in an accident.) Furthermore, $(B \varphi \rightarrow B K \varphi)$ models the attitude of an agent who thinks (believes) that he is very critical in adopting beliefs: he only believes $\varphi$ if he believes that he knows $\varphi$.

However, $(B \varphi \rightarrow B K \varphi)$ is not a property of all notions of belief. For instance, we can imagine a mathematician believing Fermat's theorem is true, without believing that he knows it is true. Moreover, $\neg(B \varphi \rightarrow B K \varphi)$ might be satisfiable in systems that interpret belief as a 'practical, working belief'. If I leave home on a bright day, I may adopt the working belief that it will not rain that day (so leave my raincoat at home), although I need not believe that I know that it will stay dry. Also, it seems that, if $B$ is interpreted as some religious belief, $(B \varphi \rightarrow B K \varphi)$ 
need not hold: $(B \varphi \wedge \neg B K \varphi)$, even $(B \varphi \wedge B \neg K \varphi)$ seems perfectly consistent then.

Negative introspection formula $(\neg X \varphi \rightarrow Y \neg Z \varphi)$ has, if $(K \varphi \rightarrow B \varphi)$ is valid, $(\neg K \varphi \rightarrow$ $K \neg B \varphi)$ as its strongest instantiation, and $(\neg B \varphi \rightarrow B \neg K \varphi)$ as its weakest. Negative introspection is closely related to the problem of 'awareness' (cf. $[5,15]$ ). $\neg X \varphi$ could be true because the agent is not aware of $\varphi$. Now, if $Y$ is the belief or knowledge of the same agent, and $(\neg X \varphi \rightarrow Y \neg Z \varphi)$ is true, he becomes aware of $\varphi$. Note that $\mathrm{A} 3(\neg K \varphi \rightarrow K \neg K \varphi)$ is a strong property of knowledge: by contraposition, it implies, that the agent's ignorance of his ignorance is sufficient to have knowledge: $(\neg K \neg K \varphi \rightarrow K \varphi)$.

The following theorem says that $K B$ is saturated with respect to the classes of $I T$ (cf. Definition 6.1).

THEOREM 6.3

$K B$ is maximal in the sense that adding any introspection, extraspection or trust formula to it makes $B_{i} \varphi \leftrightarrow K_{\imath} \varphi$ a theorem.

PROOF. We carry out the proof for the classes of introspection formulas; the other cases are similar. Due to the previous theorem, in each class we can find some 'weakest' formulas that are not $K B$. theorems yet. The weakest p.i.- formula outside $K B$ is $B_{2} \varphi \rightarrow B_{2} K_{i} \varphi$ (cf. Theorem 4.6). We have seen (in Section 5) that indeed $K B \cup\left\{B_{i} \varphi \rightarrow B_{i} K_{i} \varphi\right\} \vdash B_{i} \varphi \rightarrow K_{i} \varphi$. Or, for the case of negative introspection, we know from Fig. 3 that $\neg K_{\imath} \varphi \rightarrow B_{i} \neg K_{\imath} \varphi$ is the weakest non-theorem of $K B$ in this class. Since, by $\mathrm{T} 5, B_{i} \neg K_{i} \varphi$ is equivalent to $\neg B_{\imath} \varphi$, we immediately have $K B \cup\left\{\neg K_{\imath} \varphi \rightarrow B_{1} \neg K_{1} \varphi\right\} \vdash B_{i} \varphi \rightarrow K_{i} \varphi$.

Now we have some more equipment to look at our BCB-problem again.

\section{DEFINITION 6.4}

Let $K B^{+}$be the system $K B \backslash\{A 3\}$ together with:

$$
\begin{array}{ll}
A 3^{\prime} & K_{i} \varphi \rightarrow K_{i} K_{i} \varphi \\
A 1^{+} & B_{i} B_{i} \varphi \rightarrow B_{i} \varphi \\
A 2^{+} & B_{i} \neg B_{i} \varphi \rightarrow \neg B_{i} \varphi \\
A 3^{+} & B_{i} \varphi \rightarrow B_{i} K_{i} \varphi \\
A 4^{+} & \left.\neg B_{i} \varphi \rightarrow K_{i}\right\urcorner B_{i} \varphi \\
A 5^{+} & \left.\neg K_{i} \varphi \rightarrow B_{i}\right\urcorner K_{i} \varphi .
\end{array}
$$

\section{REMARK 6.5}

Here, we will not discuss whether $K B^{+}$models some interesting notion of belief and knowledge. Technically, we can relate $K B^{+}$with $K B$ in terms of the notions developed in this section. The basic idea behind $K B^{+}$is that it solves the BCB-problem and is quite similar to $K B$. An important reference for this similarity is $I T$. In Definition 6.4 we take benefit of the nice order in each of the $I T$-classes. We defined $K B^{+}$such that it has the same $I T$-properties as $K B$, with $\left(B_{i} \varphi \rightarrow B_{i} K_{\imath} \varphi\right)$ added to it, and formulas that yield $\left(B_{2} \varphi \rightarrow K_{i} \varphi\right)$ left out. For instance, for the class $P E$, we take care that $K B^{+}$lacks $B_{i} K_{i} \varphi \rightarrow K_{i} \varphi$ (it would yield, using $A 3^{+}$, $B_{i} \varphi \rightarrow K_{i} \varphi$ ), and add $A 1^{+}$, which is similar to $K B^{\prime}$ s Theorem T6. The models for $K B^{+}$are understood by applying Theorem 4.3. Then, in the same way as in 4.6 , non-theorems of $K B^{+}$ can be found.

The next theorem compares the two systems with respect to $I T$. In particular, item 6 of Theorem 6.6 states that we cannot make $K B$ and $K B^{+}$look more alike with respect to $I T$. Lemma 6.7 shows that outside IT, $K B$ and $K B^{+}$can still differ. 


\section{THEOREM 6.6}

$K B^{+}$satisfies the following properties:

1. $K B^{+} \vdash B_{\imath} \varphi \rightarrow B_{i} K_{i} \varphi$ and $K B^{+} \not B_{i} \varphi \rightarrow K_{i} \varphi$.

2. For all axioms $\chi$ of $K B$ such that $\chi \notin I T$ :

$$
K B^{+} \vdash \chi
$$

3. For all axioms $\chi$ of $K B^{+}$such that $\chi \notin I T$ :

$$
K B \vdash \chi
$$

4. For all $\chi \in I T \backslash\left\{B_{i} \varphi \rightarrow B_{i} K_{i} \varphi\right\}$

$K B^{+} \vdash \chi \Rightarrow K B \vdash \chi$

5. For all $\chi \in I T \backslash\left\{\neg K_{1} \varphi \rightarrow K_{2} \neg K_{\imath} \varphi, B_{i} K_{i} \varphi \rightarrow K_{2} \varphi\right\}$ :

$K B \vdash \chi \Rightarrow K B^{+} \vdash \chi$

6. For all $\chi \in I T, K B^{\prime} \in\left\{K B, K B^{+}\right\}$:

$\left(K B^{\prime} \vdash \chi\right.$ or $\left.K B^{\prime} \cup\{\chi\} \vdash B_{i} \varphi \rightarrow K_{i} \varphi\right)$

PROOF. The first part of item 1 follows by definition of $\mathrm{KB}^{+}$, the second part can be verified by finding a model for $\mathrm{KB}^{+}$(the structure of this model is immediately read off from Definition 6.4, together with Theorem 4.3) for which $S_{1} q T_{i}$. Items 2 and 3 are true by definition of $K B^{+}$. Finally, 4, 5 and 6 are easily verified by checking them for the strongest formula $\chi$ in each class (cf. Theorem 6.2) for which the antecedent is true (in case of 5 and 6).

\section{LEMMA 6.7}

$K B^{+} \vdash B_{i}\left(B_{i} \varphi \rightarrow K_{i} \varphi\right)$, but $K B \nvdash B_{i}\left(B_{i} \varphi \rightarrow K_{i} \varphi\right)$.

Solving the BCB-problem boils down to investigating the possibility of having certain combinations of $I T$-formulas. Of course, one can do this independently from the BCB-problem and study what kind of $K B$-like systems are possible anyhow. For instance, one might want a modal system modelling knowledge and belief of two agents $\left(K B_{2}\right)$. Then, one might assume maximal p.i. properties (in $K B_{2}$, this amounts to $K_{h} \varphi \rightarrow K_{i} K_{j} \varphi, h, i, j \in\{1,2\}$ : if one agent knows $\varphi$, they both know that they both know $\varphi$ ) and wonder what properties can be added to them, without implying a collapse of both operators. We end this section with a theorem about possible combinations.

THEOREM 6.8

Consider the following 'extreme systems' $P I, N I, P E$ and $N E$, which are systems with two epistemic operators $B$ and $K$ satisfying $\mathrm{A} 0, \mathrm{R} 0, \mathrm{R} 1$ (for $K$ ), $\mathrm{A} 1$ and $\mathrm{A} 8$ of $K B$, and such that:

- in $P I$ all instantiations of positive introspection are valid

- in $N I$ all instantiations of negative introspection are valid

- in $P E$ all instantiations of positive extraspection are valid

- in $N E$ all instantiations of negative extraspection are valid.

Then (in the following, the variables $X, Y, Z$ range over $\{K, B\}$ and $K^{\prime}=B$, whereas $B^{\prime}=K$ ): 
1. for any $T \in\{P I, N I, P E, N E\}, T \forall K \varphi \rightarrow B \varphi$ and $T \forall B \varphi \rightarrow K \varphi$

2. $S \nvdash K \varphi \rightarrow B \varphi$ and $S \forall B \varphi \rightarrow K \varphi$, both for $S=P I \cup N E$ and $S=N I \cup P E$.

3. adding p.e. formula $X Y \varphi \rightarrow Z \varphi$ to $P I$ yields $Z^{\prime} \varphi \rightarrow Z \varphi$

4. adding n.e. formula $X \neg Y \varphi \rightarrow \neg Z \varphi$ to $N I$ yields $Z \varphi \rightarrow Z^{\prime} \varphi$

5. adding p.i. formula $X \varphi \rightarrow Y Z \varphi$ to $P E$ yields $X \varphi \rightarrow X^{\prime} \varphi$

6. adding n.i. formula $\neg X \varphi \rightarrow Y \neg Z \varphi$ to $N E$ yields $X^{\prime} \varphi \rightarrow X \varphi$

7. adding n.i. formula $\neg X \varphi \rightarrow Y \neg Z \varphi$ to $P I$ yields $\left(\neg Y\right.$ false $\left.\rightarrow\left(X^{\prime} \varphi \rightarrow X \varphi\right)\right)$

8. adding p.i. formula $X \varphi \rightarrow Y Z \varphi$ to $N I$ yields $\left(\neg Y\right.$ false $\left.\rightarrow\left(X \varphi \rightarrow X^{\prime} \varphi\right)\right)$

9. adding n.e. formula $X \neg Y \varphi \rightarrow \neg Z \varphi$ to $P E$ yields $\neg X$ false

10. adding p.e. formula $X Y \varphi \rightarrow Z \varphi$ to $N E$ yields $\neg X$ false

11. adding $\neg X$ false to $P I$ yields the four n.e. formulas $X \neg Y \varphi \rightarrow \neg Z \varphi$

12. adding $\neg X$ falseto $N I$ yields the four p.e. formulas $X Y \varphi \rightarrow Z \varphi$.

Proof. As an example, we prove 1, 2, 3, 6, 9 and 11 .

1. $T=P I$, construct a frame such that $R^{1}$ is transitive over $\left(R^{2}, R^{3}\right)$, for all $R^{1}, R^{2}, R^{3} \in$ $\{S, T\}$, but $(S \nsubseteq T)$ and $(T \notin S)$; for instance, $W=\{v, w\}, S=\{(v, v)\}, T=$ $\{(w, w)\}$.

2. For $S=P I \cup N E$, Let $\mathcal{F}=\langle W, S, T\rangle$, with $W=\{t, u, v, w\}, S=\{(t, u),(t, w)$, $(u, w),(v, w),(w, w)\}$ and $T=\{(t, v),(t, w),(u, w),(v, w),(w, w)\} . \mathcal{F}$ is an $S$-frame, but $\mathcal{F} \not \models K \varphi \rightarrow B \varphi$ and $\mathcal{F} \not \models B \varphi \rightarrow K \varphi$.

3. Suppose we add the formula $X Y \varphi \rightarrow Z \varphi$ to $P I$. Then immediately: $Z^{\prime} \varphi \Rightarrow_{P I} X Y \varphi \rightarrow$ $Z \varphi$.

6. $X^{\prime} \varphi \Rightarrow_{p . i .} Y Z \varphi \Rightarrow \neg Y$ false $\neg Y \neg Z \varphi \Rightarrow_{\text {added n.2.-formula }} X \varphi$.

9. $X$ false $\Rightarrow X Y \varphi \wedge X \neg Y \varphi \Rightarrow_{\text {p.i. }} Z \varphi \wedge X \neg Y \varphi \Rightarrow_{\text {added n.e.-formula }} Z \varphi \wedge \neg Z \varphi \Rightarrow$ false.

11. $\neg X$ false $\Rightarrow(X \neg Y \varphi \rightarrow \neg X Y \varphi) \Rightarrow_{\text {p.1. }}(X \neg Y \varphi \rightarrow \neg Z \varphi)$.

REMARK 6.9

Theorem 6.8 has many implications. For instance, it follows from 1, 3, 7 and 11, although it is possible to have a system with two maximally p.i.-related operators, adding one p.e. instantiation to it gives either $K \varphi \rightarrow B \varphi$ or $B \varphi \rightarrow K \varphi$. The same holds for adding an n.i.-formula, provided that $\neg Y$ false holds for a suitable $Y$. Moreover, if we assume the latter, all n.e.formulas are imported to the theory. Theorem 6.8 shows an asymmetry between systems with maximal introspection, and those with maximal extraspection. For example, adding n.e.-formula $X \neg Y \varphi \rightarrow \neg Z \varphi$ to $P E$ does not yield $\left(\neg X\right.$ false $\left.\rightarrow\left(Z \varphi \rightarrow Z^{\prime} \varphi\right)\right)$, it just gives $X Y \varphi \Rightarrow_{P E}$ $Z \varphi \Rightarrow \neg X \neg Y \varphi$.

\section{Conclusions and problems}

Studying the BCB-problem, I applied some correspondence theory to multi-modal epistemic logic. Studying this multi-modal system, possible combinations of epistemic properties could be examined systematically. With this general approach I showed that Kraus and Lehmann's $K B$ is saturated with respect to many important properties (such as introspection): adding any of them to $K B$ yields $B_{i} \varphi \leftrightarrow K_{i} \varphi$. I investigated one of the many possible systems that are 'close to 
$K B^{\prime}$ and that solves the BCB-problem. This shows that the collapse of knowledge and belief one obtains by adding $B \varphi \rightarrow B K \varphi$ is not caused by the use of Kripke semantics. I argued that the techniques presented in this paper can straightforwardly be applied to probabilistic Kripke structures as well.

By allowing more epistemic operators (for each agent), many notions of belief can be combined. It seems interesting to explore this idea of having a spectrum of beliefs, ranging from weak belief, corresponding with having less alternatives (worlds) in the structure (cf. [15], where a notion of belief is defined as a possibility operator) to knowledge as some 'limit'. This idea might be extended to do a kind of 'quantitative reasoning' as follows. With respect to a relation $R$, define operators $L_{n}, n \in \mathbb{N}$, with interpretation of $L_{n} \varphi$ : 'in all, except for at most $n$ worlds, $\varphi$ is the case'. This enables defining notions like ' $\varphi$ is believed at least as strong as $\psi$ '. At the moment, I are studying some interesting perspectives offered by this option. The idea of having such 'numerical' modal operators was suggested independently in [6] and [11]. A first application to epistemic logic is to be found in [16].

\section{Acknowledgements}

I am thankful to John-Jules Meyer for the fruitful discussions we had, and for his encouraging ideas. Gerard Vreeswijk read a preliminary version of this report. Maarten de Rijke and two anonymous referees gave valuable suggestions which amended this paper in several places.

\section{References}

[1] J. F. A. K. van Benthem. Modal correspondence theory. PhD thesis, Instituut voor Grondslagenonderzoek, University of Amsterdam, 1976.

[2] J. F. A. K. van Benthem. Madal Logic and Classical Logic. Bibliopolis, Naples, 1983.

[3] C. Chang and H. Keisler. Model Theory. North Holland, Amsterdam, 1973.

[4] B. F. Chellas. Modal Logic, an Introduction. University Press, Cambridge, 1980.

[5] R. F. Fagin and J. Y. Halpern. Belief, awareness, and limited reasoning. Artificial Intelligence 34, 39-76, 1988.

[6] M. Fattorosi Barnaba and F. de Caro. Graded modalities i. Studia Logica 44, 197-221, 1985.

[7] R. Goldblatt. Logics of Time and Computation. Number 7 in CSLI Lecture Notes. Standford University, Stanford, 1987.

[8] J. Y. Halpern. The relationship between knowledge, belief and cerainty. Annals of Mathematics and Arificial Intelligence 4, 301-322, 1991.

[9] J. Y. Halpem and Y. O. Moses. A guide to the modal logies of knowledge and belief. In Proceedings IJCAI-85, pp. 480-490, Los Angeles, CA, 1985.

[10] J. Hintikka. Knowledge and Belief. Comell University Press, Ithaca, NY, 1962.

[11] W. van der Hoek. On the semantics of graded modalities. Technical Report IR-246, Free University of Amsterdam, 1991. To appear in The Joumal of Applied Non Classical Logics.

[12] W. van der Hoek. Qualitative modalities. In Proceedings of SCAl-91, Roskilde, Denmark, ed. B. Mayoh, Pp. 322-327. Ansterdam, 1991. IOS Press.

[13] W. van der Hoek. Modalities for reasoning abour knowledge and quantities. PhD thesis, Free University of Amsterdan, 1992.

[14] W. van der Hoek and J.-J. Ch. Meyer. Making some issues of implicit knowledge explicit. To appear in Foundarions of Computer Science.

[15] W, van der Hoek and J.-J. Ch. Meyer. Possible logics for belief. Technical Report IR-170, Free University of Amsterdam, 1988. To appear in Logique et Analyse.

[16] W. van der Hoek and J.-J. Ch. Meyer. Graded modalities for episternic logic. Technical Report IR 261, Free University Amsterdam, 1991. To appear in LNCS 1992. 
[17] W. van der Hoek and J.-J. Ch. Meyer. A model-theoretic study of knowledge. Technical Report IRXXX, Free University Amsterdam, 1992.

[18] G. E. Hughes and M. J. Cresswell. Intraducrion to Modal Logic. Methuen, London, 1968.

[19] S. Kraus and D. Lehmann. Knowledge, belief and time. In Proceedings ICALP, number 226 in Lecture Notes in Computer Science, ed. L. Knott. Springer, 1986. Extended version in Theoretical Computer Science 58, 155-174 1988.

[20] G. Lokemeyer. Steps towards a first-order logic of explicit and implicit belief. In J.Y. Halpern, editor, Proceedings of TARK Conference, pages 325-340, 1988.

[21] K. Lehrer and T. D. Paxson. Knowledge: Undefeated justified true belief. The Joumal of Philosophy 66, 225-237, 1969.

[22] W. Lenzen. Glauben, Wissen und Warscheinlichkeit. Springer Verlag, Wien, 1980.

[23] J.-J. Ch. Meyer, W. van der Hoek, and G. A. W. Vreeswijk. Epistemic logic for computer science: A tutorial, Part I. EATCS Bulletin 44, 242-270, 1991.

[24] J.-J. Ch. Meyer, W. van der Hoek, and G. A. W. Vreeswijk. Epistemic logic for computer science: A tutorial, Part II. EATCS Bulletin 45, 256-287, 1991 (part II).

[25] M. de Rijke. Correspondence theory of modal logic. Technical Report, ILC, Amsterdam, 1993.

[26] H. Sahlquist. Completeness and correspondence in the first- and second-order semantics for modal logic. In Proceedings of the 3rd Scandanavian Logic Symposium, ed. S. Kanger. North Holland, 1975.

[27] Y. Shoham and Y. Moses. Belief as defeasible knowledge. In Proceedings Eleventh Intemational Joint Conference on Arificial Intelligence (IJCAI), pp. 1168-1173, 1989.

[28] F. Voorbrak. The logic of objective knowledge and rational belief. In Logics in Al, number 478 in Lecture Notes in Artificial Intelligence, ed. J. van Eijck, pp. 499-516. Springer, 1990.

Received 22 January 1991 